\title{
A self-consistent model of a $22 \mathrm{GHz}$ water maser in a dusty environment near late-type stars
}

\author{
N. Babkovskaia and J. Poutanen
}

Astronomy Division, PO Box 3000, 90014 University of Oulu, Finland

e-mail: [natalia.babkovskaia; juri.poutanen]@oulu.fi

Received 9 June 2005 / Accepted 5 October 2005

\section{ABSTRACT}

\begin{abstract}
We study the conditions for operation of the $22 \mathrm{GHz}$ ortho-water maser in a dusty medium near late-type stars. The main physical processes, such as exchange of energy between dust and gas in the radiation field of a star, radiative cooling by water molecules and pumping of water masers are described self-consistently. We show that the presence of dust grains of various types (or of one type with size distribution) strongly affects the maser action. The pumping mechanism based on the presence of the dust of different optical properties is able to explain water masers in the silicate carbon star V778 Cyg. However, the masers in the winds from asymptotic giant branch stars require an additional source of heating, for instance due to the dust drift through the gas.
\end{abstract}

Key words. masers - radio lines: general - stars: AGB and post-AGB - stars: late-type - methods: numerical - dust, extinction

\section{Introduction}

$22 \mathrm{GHz}\left(6_{16} \rightarrow 5_{23}\right)$ ortho-water maser emission has been detected from many astronomical objects, such as late-type stars, star formation regions, and active galactic nuclei. In recent years, much progress in studying maser objects has been made due to increasing angular resolution and sensitivity of radiointerferometers, which allow one to obtain detailed maps of the distribution of maser sources. These observations provide important information about the structure, physical conditions and kinematics of the gas in these sources. The observational data show that in most of them, maser radiation comes from the region co-spatial with sources of the infrared dust emission (Downes et al. 1981; Yamamura et al. 2000; Danchi et al. 1994) and, therefore, maser amplification may take place in a dusty environment.

In asymptotic giant branch stars (AGBs) and red supergiants (RSGs), masers are mostly observed in stellar winds. Water masers observed in some silicate carbon stars, e.g. V778 Cyg (Engels 1994; Engels \& Leinert 1994), are not associated with the winds, but most probably arise in an oxygenrich disk around a companion star in a binary system (Szczerba et al. 2005).

The first model of water masers in late-type stars was proposed by Deguchi (1977), who considered an expanding, spherically symmetric circumstellar envelope. The line photons were assumed to escape from the wind due to the high velocity gradients. The gas temperature was not computed self-consistently, but was assumed to be a power-law function of distance from the star. In his model, the strongest water masers are predicted along tangential directions where the coherent length is largest. He used approximate formulae for the collisional cross-sections and neglected the influence of dust on the maser strength.

Cooke \& Elitzur (1985) have presented a more elaborate model employing a new set of collisional cross-sections by Green (1980) and using the wind parameters taken from the model of Goldreich \& Scoville (1976). In this model, the acceleration of the wind is provided by the radiation pressure on the dust particles, the temperature was assumed to be determined by the stellar radiation, while the gas is viscously heated by collisions with faster moving dust grains. Cooke \& Elitzur (1985) assume the same temperature profile for outflows with very different characteristics. They have accounted for the influence of dust emission (using a simplified dust absorption coefficient) on the excitation of water molecule, but ignored the effect of line photon absorption by the dust. The escape of photons was again assumed to be determined by the velocity gradient.

Humphreys et al. (2001) also simulated masers in the winds in a Sobolev (1960) approximation, using more recent collisional cross-sections provided by Green et al. (1993). They obtained, however, suspiciously high gas temperatures because of ignoring molecular cooling. The influence of dust absorption on the water molecule population was again neglected.

Recent observations by MERLIN and VLA have shown that the winds from AGBs and RSGs are strongly inhomogeneous. Water masers arise at distances of 10-60 AU from AGBs 
and have a typical size of 2-4 AU across (Bains et al. 2003), while in RSGs all sizes are 5 to 10 times larger. Moreover, the filling factor of the clouds is only $\sim 0.01$. Because of the relatively small sizes, the velocity gradient within the clouds is negligible and the Sobolev approximation becomes questionable.

The aforementioned maser wind models (Deguchi 1977; Cooke \& Elitzur 1985; Humphreys et al. 2001) operate on the de Jong (1973) mechanism of collisional pumping where the breakdown of thermal equilibrium occurs because the gas becomes transparent for different transitions at different physical depths. However, the presence of dust can strongly influence the maser strength (Goldreich \& Kwan 1974; Kegel 1975; Strelnitskij 1977; Bolgova et al. 1977). Goldreich \& Kwan (1974) suggested that the hot dust radiation can excite water molecules to the vibrational state, and the heat sink could be provided by collisions with cooler (than dust) hydrogen molecules. The possibility of inversion in such a situation was questioned by Deguchi (1981). Alternatively, the cold dust can produce the necessary inversion (Strelnitskij 1977; Bolgova et al. 1977; Deguchi 1981; Chandra et al. 1984a; Collison \& Watson 1995; Wallin \& Watson 1997; Yates et al. 1997; Babkovskaia \& Poutanen 2004, hereafter BP04). In an optically thick environment, the excitation temperature takes a value between the dust and the gas temperatures depending on the relative role of the dust and collisions in the destruction of the line photons. Deguchi (1981) has pointed out that water ice can provide a very effective heat sink due to a strong peak near $45 \mu \mathrm{m}$ in the absorption coefficient. For other dust types the inversion of maser level populations is smaller by just 20-30 per cent (BP04).

In the cold dust-hot gas model, an arbitrarily thick layer can participate in the maser action provided the gas and dust temperatures differ sufficiently. The temperature difference between the gas and the dust can appear in late-type stars due to the viscous gas heating by rapidly moving dust, presence of the dust particles of different types and sizes which assure their different temperatures, or shock waves. Thus, the dust present in the outflow plays a dual role, providing heating of the gas as well as absorbing line photons and increasing the maser strength. Because in this situation the photon escape is determined by dust absorption and the physical size of the maser clump, we can use the static approximation (Yates et al. 1997; BP04) for maser modeling. This model also can be used for masers in a slowly rotating disk around a companion of the silicate carbon star V778 Cyg (Szczerba et al. 2005).

The purpose of this paper is to construct a self-consistent model of water masers in the environment typical for clumpy winds from AGBs and RSGs or maser sites in the vicinity of silicate carbon stars. We take into account here the most important heating/cooling processes for the gas and dust and study in detail the influence of various dust types and sizes on the maser action. In Sect. 2, we formulate the problem and give a detailed description of calculation method. Section 3 is devoted to the results of calculations and discussion of the multi-temperature dust pumping mechanism. In Sect. 4, we illustrate our model, applying it to the masers near late-type stars. A summary is given in Sect. 5.

\section{Formulation of the problem and calculation method}

We investigate the maser effect in medium containing mixture of the gas (molecular hydrogen and water vapor) and dust in the black body radiation field of a late-type star. We simultaneously solve the population balance equation for the water molecule and the radiative transfer equation (RTE) for water spectral lines using an escape probability method. We also account for the saturation effect in masing lines. Our approach is similar to that of BP04.

In addition, we self-consistently compute the dust and gas temperature accounting for main heating and cooling processes. As we will see below, the dust temperature is determined mainly by the radiation field of a star and is independent of the processes in the gas phase, therefore it can be computed immediately.

The gas temperature, however, depends on the energy exchange with dust and cooling provided by water vapor, which depends on the level populations of the water molecule. The populations in turn depend on the gas (and dust) temperature. Therefore, we implement the iteration scheme where we first guess the gas temperature, compute populations and the corresponding cooling rate, then correct the gas temperature to satisfy the energy balance, until convergence is reached.

We consider a slab geometry (with half-thickness $H$ ) which is a reasonably good representation of clumps in the winds from AGBs and RSGs flattened in the process of formation due to shock waves and/or due to different expansion velocities in radial and tangential directions (see arguments in Bains et al. 2003). It also can be used to model masers from disks. The slab thickness $2 \mathrm{H}$ is varied from about $10^{11} \mathrm{~cm}$ to $10^{16} \mathrm{~cm}$ which is the upper limit corresponding to the total size of the masing region in RSGs (Rosen et al. 1978). The stellar temperature is taken to be $T_{*}=3000 \mathrm{~K}$. We consider the hydrogen concentration $10^{7} \lesssim N_{\mathrm{H}_{2}} \lesssim 10^{12} \mathrm{~cm}^{-3}$ and mass fraction of water $10^{-8} \lesssim f_{\mathrm{H}_{2} \mathrm{O}}=9 N_{\mathrm{H}_{2} \mathrm{O}} / N_{\mathrm{H}_{2}} \lesssim 10^{-2}$ (where $N_{\mathrm{H}_{2} \mathrm{O}}$ is water concentration and the factor of 9 comes from the ratio of water to hydrogen masses).

\subsection{Radiative transfer}

The solution of the RTE is obtained by the escape probability method. The mean intensity of the radiation averaged over the line profile at the optical depth $\tau$ (measured from the upper boundary of the slab) can be written in the form (see e.g. BP04)

$$
\begin{aligned}
& \bar{J}(\tau)=(1-p) S_{u l}+p^{\mathrm{c}} \tilde{B}, \\
& p=\delta+(1-\delta)\left[\frac{1}{2} K_{2}(\tilde{\beta}, \tau)+\frac{1}{2} K_{2}\left(\tilde{\beta}, 2 \tau_{0}-\tau\right)\right], \\
& p^{\mathrm{c}}=\delta\left[1-\frac{1}{2} L_{2}(\tilde{\beta}, \tau)-\frac{1}{2} L_{2}\left(\tilde{\beta}, 2 \tau_{0}-\tau\right)\right],
\end{aligned}
$$

where $2 \tau_{0}=\int \alpha_{\mathrm{L}} \mathrm{d} z$ is the total slab optical depth, $S_{u l}=$ $\left(\frac{n_{l}}{n_{u}} \frac{g_{u}}{g_{l}}-1\right)^{-1}$ is the line source function (all intensities are measured in units $\left.2 h v_{u l}^{3} / c^{2}\right), v_{u l}$ is the transition frequency, $n_{u}$ and $n_{l}$ are the fractional level populations, $g_{u}$ and $g_{l}$ are the statistical weights for the upper and lower levels, respectively, $\tilde{B}$ is the 
dust source function (Planck function), $\beta=\alpha_{\mathrm{d}} / \alpha_{\mathrm{L}}$ is the ratio of the absorption coefficients due to the dust and the line, $\delta(\beta)$ is the probability per single interaction that the line photon will be absorbed by dust, $K_{2}$ is the probability that the line photon will escape from the slab and $L_{2}$ is the probability that the photon emitted by the dust will escape from the slab. We use approximations of $\delta, K_{2}$, and $p^{\mathrm{c}}$ from BP04. In most of our calculations we take $\tau=\tau_{0}$ (i.e. slab center). The line absorption coefficient is

$\alpha_{\mathrm{L}}=\lambda_{u l}^{3} g_{u} A_{u l} \Delta n_{u l} N_{\mathrm{H}_{2} \mathrm{O}} \frac{v_{u l}}{8 \pi c \Delta v_{\mathrm{D}}}$.

Here, $\Delta n_{u l}=n_{l} / g_{l}-n_{u} / g_{u}, \Delta v_{\mathrm{D}} / v_{u l}=\left(2 k T / 18 m_{\mathrm{p}} c^{2}\right)^{1 / 2}$ is the Doppler width, $A_{u l}$ is the Einstein coefficient, $T$ is the gas kinetic temperature, $m_{\mathrm{p}}$ is the proton mass and $\lambda_{u l}=c / v_{u l}$.

\subsection{Masing transitions}

Transitions with inverted level populations (masing lines) need a different treatment. We neglect the dust influence on masing transitions, i.e. assume $\beta=\delta=p^{c}=0$ (e.g. $|\beta| \sim 10^{-4}$ for the $6_{16} \rightarrow 5_{23}$ transition). The mean intensity of the radiation in the $u l$-line in the slab center is

$J\left(\tau_{x}\right) \simeq S_{u l} \int_{0}^{1}\left[1-\exp \left(-\tau_{x} / \mu\right)\right] \mathrm{d} \mu$,

where $\mu$ is the cosine of the angle between the direction of propagation and the outward normal, $\tau_{x}=\tau_{\mathrm{m}} \phi_{x}, \tau_{\mathrm{m}}=\sqrt{\pi} \tau_{\mathrm{c}}=$ $\alpha_{\mathrm{L}}^{\mathrm{m}} H$ is the optical depth in the maser line, $\tau_{\mathrm{c}}$ is the optical depth in the line center, $\alpha_{\mathrm{L}}^{\mathrm{m}}$ is the maser absorption coefficient, $x=\left(v-v_{u l}\right) / \Delta v_{\mathrm{D}}$ is the frequency within the spectral line in thermal Doppler units, $\phi_{x}=\pi^{-1 / 2} \exp \left(-x^{2}\right)$ is the Doppler profile. In the case of inversion, the optical depth is negative and the mean intensity grows to infinity. This happens because the path length parallel to the boundary of the slab $(\mu=0)$ becomes infinite. In a real astrophysical system such a singularity does not appear because the coherent amplification length is limited by the size of the maser source (or velocity gradients). If we approximate the geometry by a cylinder with half-height $H$ and radius $L$, Eq. (3) can be rewritten in the form

$$
\begin{aligned}
J\left(\tau_{x}\right) \simeq & S_{u l} \int_{0}^{\mu_{\min }}\left[1-\exp \left(-\tau_{x} / \mu_{\min }\right)\right] \mathrm{d} \mu \\
& +S_{u l} \int_{\mu_{\min }}^{1}\left[1-\exp \left(-\tau_{x} / \mu\right)\right] \mathrm{d} \mu,
\end{aligned}
$$

where $\mu_{\min }=H / L$. The first integral on the right hand side is $\mu_{\min }\left[1-\exp \left(-\tau_{x} / \mu_{\min }\right)\right]$, while the second one is approximately $\left[1-\mu_{\min }-\mu_{\min }^{2} /\left|\tau_{\mathrm{c}}\right| \exp \left(-\tau_{x} / \mu_{\min }\right)\right]$, when $\left|\tau_{x} / \mu_{\min }\right| \gg 1$. Therefore, for large $\left|\tau_{x} / \mu_{\min }\right|$ the mean intensity in the maser line is

$J\left(\tau_{x}\right) \simeq S_{u l}\left[1-\mu_{\min } \exp \left(-\tau_{x} / \mu_{\min }\right)\right]$.

At large $\left|\tau_{x} / \mu_{\min }\right|, J_{x}$ grows and the masing transition starts affecting level populations (the maser starts to saturate). The critical value of $\tau_{\mathrm{m}}$ at which the maser becomes saturated depends on the geometry of the maser source, and thus on the value of $\mu_{\min }$. The case of $\mu_{\min }=1$ corresponds to an almost spherical maser, while $\mu_{\min } \ll 1$ corresponds to a flat configuration, e.g. a shock wave or an accretion disk.

The line-averaged intensity $\bar{J}=\int \phi_{x} J\left(\tau_{x}\right) \mathrm{d} x$ in maser lines is given by Eq. (1), where $K_{2}$ should be replaced by

$K_{2}^{\mathrm{m}}=\int_{-\infty}^{\infty} \phi_{x} \mu_{\min } \exp \left(-\tau_{x} / \mu_{\min }\right) \mathrm{d} x$.

One can show that

$K_{2}^{\mathrm{m}} \simeq \frac{\mu_{\min } \exp \left(\left|\tau_{\mathrm{c}}\right| / \mu_{\min }\right)}{\left|\tau_{\mathrm{c}}\right| \sqrt{\pi \ln \left|\tau_{\mathrm{c}}\right|}}$, if $\left|\tau_{\mathrm{c}}\right| \rightarrow \infty$

while for $\left|\tau_{\mathrm{c}}\right| \rightarrow 0, K_{2}^{\mathrm{m}}$ must smoothly approach $K_{2}$ for nonmasing transitions (Hollenbach \& McKee 1979; Babkovskaia $\&$ Poutanen 2004). We propose here a formula that connects these two asymptotes (it generalizes Eq. (18) in BP04 to $\left.\mu_{\min }<1\right)$ :

$K_{2}^{\mathrm{m}}=\frac{\mu_{\min } \exp \left(\left|\tau_{\mathrm{c}}\right| / \mu_{\min }\right)+1-\mu_{\min }+1.18\left|\tau_{\mathrm{c}}\right|}{1+\left|\tau_{\mathrm{c}}\right|\left[\pi \ln \left(1+\left|\tau_{\mathrm{c}}\right|\right)\right]^{1 / 2}}$.

The smoothness of $K_{2}$, when $\tau$ passes through zero, allows us to account for the effect of maser saturation.

To characterize the maser strength we use the optical depth $\tau_{\mathrm{m}} \equiv \alpha_{\mathrm{L}}^{\mathrm{m}} H$ determined along the axis of the cylinder. This quantity characterizes only local conditions in the slab center. With increasing $H$, the maser may stop operating in the slab center, reducing such computed $\tau_{\mathrm{m}}$. This, however, just means that maser may operate more efficiently away from the central plane closer to the slab boundary.

For a cylindrical maser, the actual observed intensity is larger along the radius of cylinder than along its axis. To estimate the maximum power of unsaturated cylindrical masers, one should use the corresponding optical depth:

$\tau_{\max }=(2 L / H) \tau_{\mathrm{m}} / \sqrt{\pi}=2 L \alpha_{\mathrm{L}}^{\mathrm{m}} / \sqrt{\pi} \approx \tau_{\mathrm{m}} / \mu_{\min }$.

If the maser is unsaturated (i.e. the maser radiation does not affect the level populations), the absorption coefficient in a masing line does not depend on $\mu_{\text {min }}$. However, the optical thickness along the line of sight and, therefore, the maser luminosity do depend on $\mu_{\mathrm{min}}$, which is determined by the geometry of the source. For simplicity of calculations, we assume $\mu_{\min }=1 \mathrm{ev}-$ erywhere except an application in Sect. 3.5.1.

\subsection{Population balance}

Using the solution of the RTE in the escape probability approximation (1), the statistical balance equations take the form (see e.g. BP04)

$$
\begin{aligned}
& \sum_{l>u} A_{l u}\left(p_{l u} n_{l}+p_{l u}^{\mathrm{c}} \tilde{B}_{l u} g_{l} \Delta n_{u l}\right)-\sum_{l<u} A_{u l}\left(p_{u l} n_{u}+p_{u l}^{\mathrm{c}} \tilde{B}_{u l} g_{u} \Delta n_{l u}\right) \\
& =\sum_{l \neq u}\left(C_{u l} n_{u}-C_{l u} n_{l}\right), \quad u=1,2, \ldots, M-1
\end{aligned}
$$

with the normalizing condition $\sum_{u} n_{u}=1$. The Einstein $A$-coefficients corresponding to the rotational transitions of the ground state are taken from Chandra et al. (1984b). Collisional 
de-excitation rates with hydrogen, $C_{u l}$, corresponding to the rotational transitions of the ground state are from Green et al. (1993), while the excitation rates are computed using the detailed balance condition. We take into account the first 45 rotational levels of the ground vibrational state and 45 rotational levels of the first-excited vibrational state (010) of ortho$\mathrm{H}_{2} \mathrm{O}$ molecules (Toth 1991) and all possible radiative and collisional transitions between them. We recalculate the Einstein coefficients of the rotational transitions in the first-excited vibration state $A_{u l}^{\mathrm{E}}$ and of the vibration-rotational transitions $A_{u l}^{\mathrm{EG}}$ from the Einstein coefficients of the rotational transitions in the ground vibrational state $A_{u l}^{\mathrm{G}}$ (Deguchi 1977)

$$
\begin{aligned}
& A_{u l}^{\mathrm{E}}=A_{u l}^{\mathrm{G}}\left(\frac{v_{u l}^{\mathrm{E}}}{v_{u l}^{\mathrm{G}}}\right)^{3}, \\
& A_{u l}^{\mathrm{EG}}=A_{u l}^{\mathrm{G}}\left(\frac{\mu^{\prime}}{\mu_{0}}\right)^{2}\left(\frac{v_{u l}^{\mathrm{EG}}}{v_{u l}^{\mathrm{G}}}\right)^{3},
\end{aligned}
$$

where $\mu^{\prime}$ is the transition dipole moment between the vibrational states and $\mu_{0}$ is the intrinsic dipole moment $\left(\left[\mu^{\prime} / \mu_{0}\right]^{2}=\right.$ $0.005), v_{u l}^{\mathrm{G}}$ is the frequency of a rotational transition in the ground vibrational state, $v_{u l}^{\mathrm{EG}}$ is the frequency of a vibrationrotational transition, $v_{u l}^{\mathrm{E}}$ is the frequency of a rotational transition in the first-excited vibrational state. Since the probabilities of the collisional transitions between vibrational states $C_{u l}^{\mathrm{EG}}$ and of the collisional rotational transitions in the first-excited vibrational state $C_{u l}^{\mathrm{E}}$ are not known, we assume, following Deguchi (1977), that

$C_{u l}^{\mathrm{EG}}=\chi C_{u l}^{\mathrm{G}}, \quad C_{u l}^{\mathrm{E}}=0$,

where $C_{u l}^{\mathrm{G}}$ is the probability of the rotational collisional transitions in the ground vibrational state, $\chi=10^{-3}$ is a fixed parameter.

The statistical balance Eqs. (10) together with Eqs. (1) for escape probabilities are solved by standard Newton-Raphson method. Our escape probability method is rather stable and allows us to account for the effect of saturation in any masing line, which is very difficult (or even impossible) to implement in an accelerated lambda iteration method (Yates et al. 1997).

\subsection{Dust properties}

We consider the following types of dust

1. amorphous water ice (Hudgins et al. 1993);

2. crystalline water ice (Bertie et al. 1969);

3. astronomical silicate (Laor \& Draine 1993);

4. circumstellar silicate (David \& Pegourie 1995);

5. graphite (Laor \& Draine 1993);

6. amorphous carbon (Rouleau \& Martin 1991).

Water ice is called "ice" hereafter. The dust optical properties can be characterized by the absorption coefficient per unit mass $K_{\mathrm{abs}, \lambda}=3 Q_{\lambda} / 4 \bar{\rho} a$, where $Q_{\lambda}$ is the absorption efficiency, $\bar{\rho}$ is the density of the dust material: $1 \mathrm{~g} \mathrm{~cm}^{-3}$ (water ice), $2.26 \mathrm{~g} \mathrm{~cm}^{-3}$ (graphite), $1.85 \mathrm{~g} \mathrm{~cm}^{-3}$ (amorphous carbon), $3.3 \mathrm{~g} \mathrm{~cm}^{-3}$ (silicates). We present $K_{\mathrm{abs}, \lambda}$ for different dust types and grain sizes as a function of the wavelength in Fig. 1.
Because of the absence of the experimental data on the optical constants for the amorphous ice at wavelength $\lambda<1 \mu \mathrm{m}$, we use data for the crystalline ice in this wavelength range instead.

We consider either the fixed size of the dust grains or a power-law distribution of the form

$\mathrm{d} n(a) \propto a^{\gamma} \mathrm{d} a, \quad a_{\min } \leq a \leq a_{\max }$,

with $a_{\min }=0.01 \mu \mathrm{m}, a_{\max }=1 \mu \mathrm{m}$, and $\gamma=-3.5$ (Mathis et al. 1977; Jura 1996). In our calculations we assume the standard dust mass-fraction for interstellar medium $f_{\mathrm{d}}=10^{-2}$. Since $\mathrm{d} f_{\mathrm{d}}=\frac{4 \pi}{3} a^{3} \bar{\rho} \mathrm{d} n(a) /\left(2 m_{\mathrm{p}} N_{\mathrm{H}_{2}}\right)$, where $\bar{\rho}$ is the density of the dust material, one can write the distribution function for the dust mass-fraction

$\mathrm{d} f_{\mathrm{d}}(a)=f_{\mathrm{d}} \frac{(\gamma+4)}{a_{\text {max }}^{\gamma+4}-a_{\text {min }}^{\gamma+4}} a^{\gamma+3} \mathrm{~d} a$.

The dust absorption coefficient is given by $\alpha_{\mathrm{d}}=K_{\mathrm{abs}} \rho_{\mathrm{d}}$, where $\rho_{\mathrm{d}}=f_{\mathrm{d}} 2 m_{\mathrm{p}} N_{\mathrm{H}_{2}}$ is the dust density. If dust of two different types is present, the dust absorption coefficient is given by the sum

$\alpha_{\mathrm{d}}=\alpha_{\mathrm{d}}^{\mathrm{c}}+\alpha_{\mathrm{d}}^{\mathrm{h}}=2 m_{\mathrm{p}} N_{\mathrm{H}_{2}}\left[K_{\mathrm{abs}}^{\mathrm{c}} f_{\mathrm{d}}^{\mathrm{c}}+K_{\mathrm{abs}}^{\mathrm{h}} f_{\mathrm{d}}^{\mathrm{h}}\right]$,

where $f_{\mathrm{d}}^{\mathrm{h}}$ and $f_{\mathrm{d}}^{\mathrm{c}}$ are the mass fractions of different dust types (the total dust mass fraction is $f_{\mathrm{d}}=f_{\mathrm{d}}^{\mathrm{h}}+f_{\mathrm{d}}^{\mathrm{c}}$ ). The dust source function is then

$\tilde{B}=\left[\alpha_{\mathrm{d}}^{\mathrm{c}} B\left(T_{\mathrm{d}}^{\mathrm{c}}\right)+\alpha_{\mathrm{d}}^{\mathrm{h}} B\left(T_{\mathrm{d}}^{\mathrm{h}}\right)\right] / \alpha_{\mathrm{d}}$,

where $T_{\mathrm{d}}^{\mathrm{h}}$ and $T_{\mathrm{d}}^{\mathrm{c}}$ are the corresponding dust temperatures. For the grain size distribution (15), the dust absorption coefficient and the source function are transformed to

$\begin{aligned} \alpha_{\mathrm{d}} & =2 m_{\mathrm{p}} N_{\mathrm{H}_{2}} \int K_{\mathrm{abs}}(a) \mathrm{d} f_{\mathrm{d}}, \\ \tilde{B} & =\frac{\int K_{\mathrm{abs}}(a) B\left[T_{\mathrm{d}}(a)\right] \mathrm{d} f_{\mathrm{d}}}{\int K_{\mathrm{abs}}(a) \mathrm{d} f_{\mathrm{d}}} .\end{aligned}$

We assume that the medium is optically thin to the absorption by dust,

$\tau_{\mathrm{d}}=2 m_{\mathrm{p}} f_{\mathrm{d}} N_{\mathrm{H}_{2}} \bar{K}_{\mathrm{abs}}\left(T_{*}\right) H \lesssim 1$,

where $\tau_{\mathrm{d}}$ is the dust optical depth, $\bar{K}_{\mathrm{abs}}$ is the Planck-averaged absorption coefficient.

\subsection{Dust cooling and heating}

The main mechanism for dust heating is provided by the stellar radiation

$q_{*}^{+}=W 8 m_{\mathrm{p}} N_{\mathrm{H}_{2}} f_{\mathrm{d}} \bar{K}_{\mathrm{abs}}\left(T_{*}\right) \sigma T_{*}^{4}$,

where $W=\frac{1}{4}\left(\frac{R}{D}\right)^{2}$ is the dilution factor, $R$ is the stellar radius, and $D$ is the distance to the star. The dust cools by its own radiation with the corresponding rate

$q_{\mathrm{rad}}^{-}=8 m_{\mathrm{p}} N_{\mathrm{H}_{2}} f_{\mathrm{d}} \bar{K}_{\mathrm{abs}}\left(T_{\mathrm{d}}\right) \sigma T_{\mathrm{d}}^{4}$.

In addition, there is energy exchange between gas and dust (Goldreich \& Kwan 1974)

$q_{\text {gas }}=N_{\mathrm{d}} A_{\mathrm{d}} N_{\mathrm{H}_{2}} V_{\mathrm{t}} k\left(T-T_{\mathrm{d}}\right)=\frac{3 m_{\mathrm{p}}}{2 \bar{\rho}} \frac{f_{\mathrm{d}}}{a} N_{\mathrm{H}_{2}}^{2} V_{\mathrm{t}} k\left(T-T_{\mathrm{d}}\right)$, 


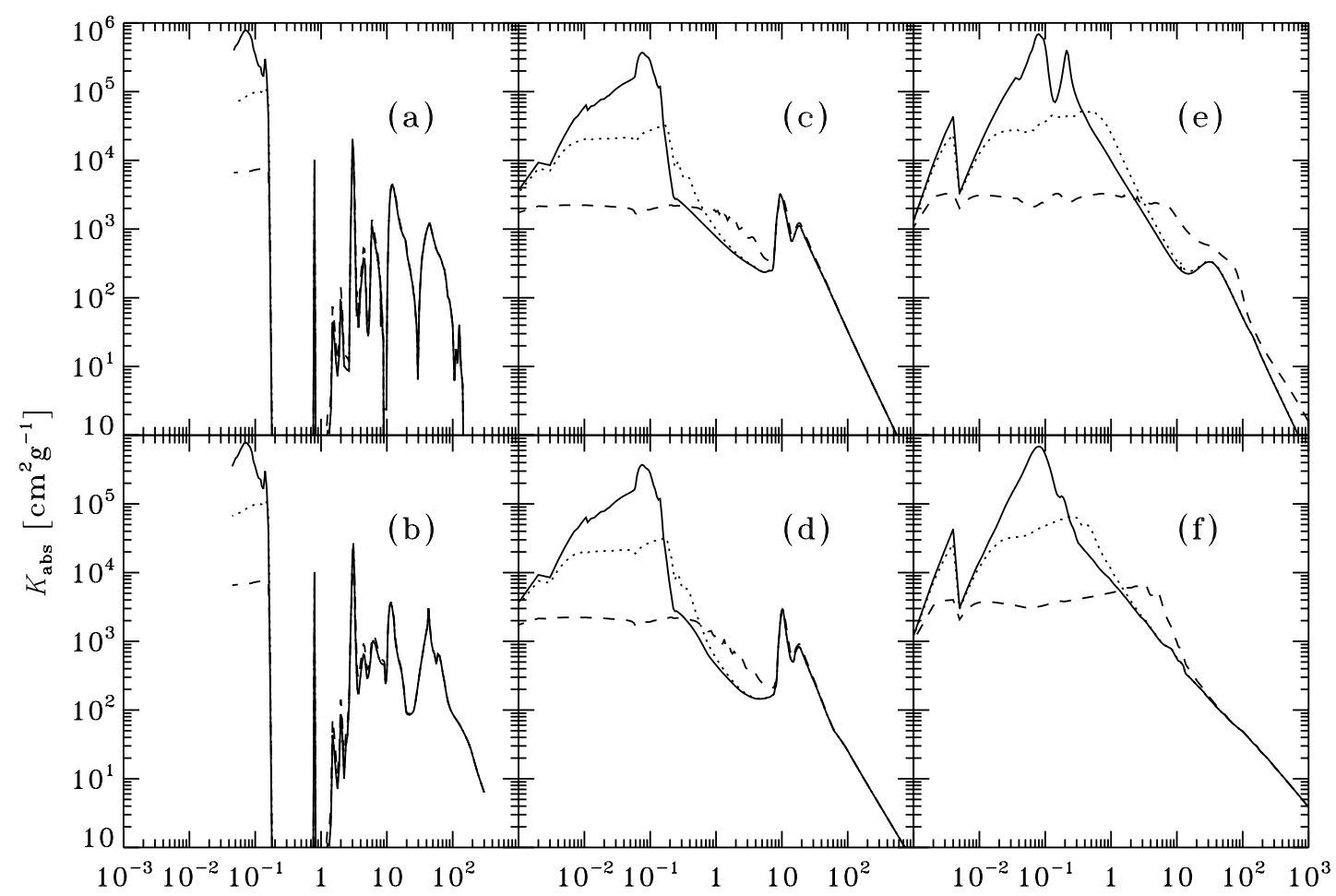

Fig. 1. Dust absorption coefficient per unit mass, as a function of the wavelength in the case of spherical dust grains of radius $a=0.01 \mu \mathrm{m}$ (solid curves), $a=0.1 \mu \mathrm{m}$ (dotted curves) and $a=1 \mu \mathrm{m}$ (dashed curves) for a) amorphous water ice (Hudgins et al. 1993), b) crystalline water ice (Bertie et al. 1969), c) astronomical silicate (Laor \& Draine 1993), d) circumstellar silicate (David \& Pegourie 1995), e) graphite (Laor \& Draine 1993), f) amorphous carbon (Rouleau \& Martin 1991).

where $N_{\mathrm{d}}$ is the concentration of the dust grains, $A_{\mathrm{d}}=\pi a^{2}$ is their geometrical cross sections, and $V_{\mathrm{t}}$ is the hydrogen thermal velocity. The energy exchange is small compared to radiative cooling and heating if $N_{\mathrm{H}_{2}} \lesssim 10^{9} \mathrm{~cm}^{-3}$ and can be neglected. Absorption of the near-infrared photons from $\mathrm{H}_{2} \mathrm{O}$ and $\mathrm{CO}$ present in the dusty medium gives an even smaller contribution to the energy exchange. The thermal balance for the dust is then transformed to a standard form

$q_{*}^{+} \simeq q_{\mathrm{rad}}^{-}$.

The dust temperatures depend only on the stellar temperature, the dilution factor (approximately as $W^{1 / 5}$ ) and the dust optical properties (see Fig. 2).

\subsection{Gas heating and cooling}

The main heating and cooling mechanisms determining the gas kinetic temperature are the dust-gas collisions, photoelectric effect on grains (de Jong 1977; Tielens \& Hollenbach 1985; Groenewegen 1994), cosmic rays (Goldsmith \& Langer 1978), viscous stresses (Shakura \& Sunyaev 1973) and radiation of $\mathrm{H}_{2} \mathrm{O}$ and $\mathrm{H}_{2}$ molecules (e.g. Goldreich \& Scoville 1976). The heating (cooling) rate of the gas per unit volume $\left(\mathrm{erg} \mathrm{cm}^{-3} \mathrm{~s}^{-1}\right)$ due to collisions with hotter (cooler) dust is given by Eq. (23):

$q_{\text {dust }}=-q_{\text {gas }}$.

The rate of gas heating due to cosmic rays and viscous stresses is several orders of magnitude smaller than that due to the

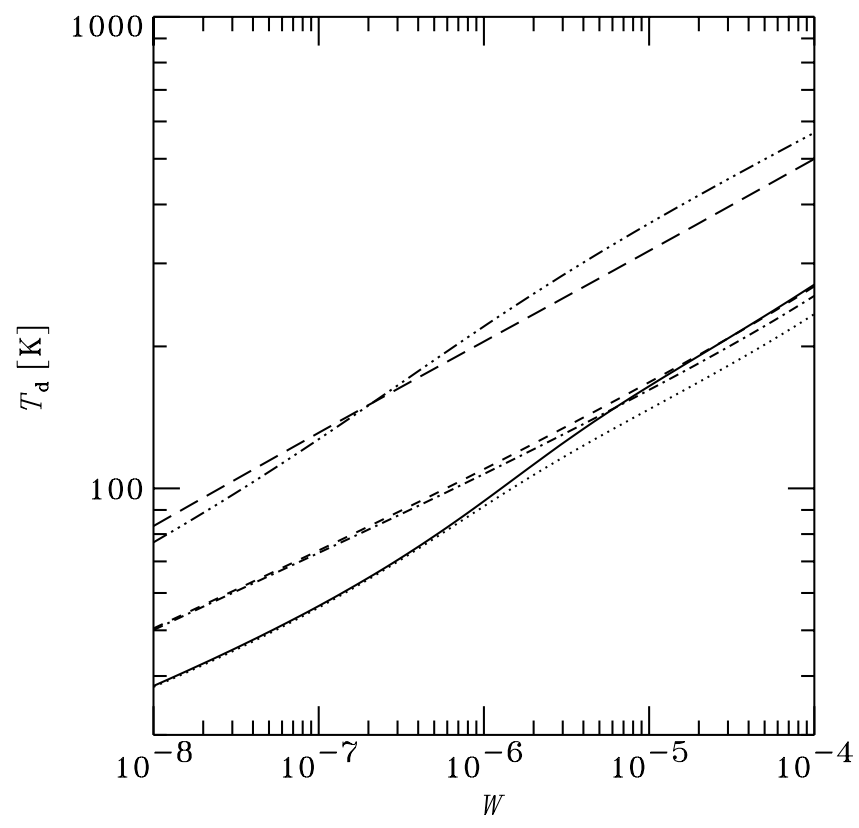

Fig. 2. Dust temperature for dust grains of radius $a=0.01 \mu \mathrm{m}$ for amorphous ice (dotted curve), crystalline ice (solid curve), astronomical silicate (dashed curve), circumstellar silicate (dot-dashed curve), graphite (triple-dot-dashed curve), amorphous carbon (long-dashed curve), as a function of the dilution factor for $T_{*}=3000 \mathrm{~K}$.

dust-gas collisions, while heating of the gas due to the photoeffect is important when the stellar radiation is strong in the UV range, and can be neglected for late-type stars. Moreover, 


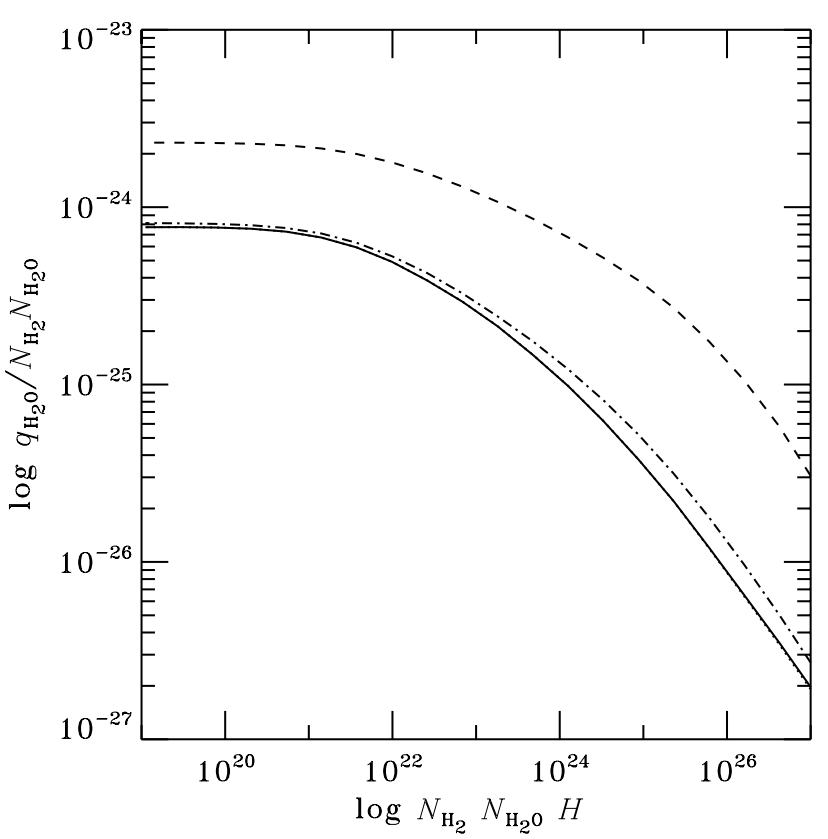

Fig. 3. Water cooling efficiency $\left(\mathrm{erg} \mathrm{cm}^{3} \mathrm{~s}^{-1}\right.$ ) as a function of $N_{\mathrm{H}_{2}} N_{\mathrm{H}_{2} \mathrm{O}} \mathrm{H}$ in the case of the water-hydrogen mixture, including the first vibrational level in water molecule: with silicate dust (solid curve) and without dust (dashed curve); taking into account only ground vibrational state: with silicate dust (dotted curve) and without dust (dotdashed curve). The dust and gas temperatures are $200 \mathrm{~K}$, the grain size is $0.01 \mu \mathrm{m}$, dust-to-gas mass ratio is $f_{\mathrm{d}}=10^{-2}$, the slab half-thickness is $H=10^{14} \mathrm{~cm}$.

in spite of the fact that hydrogen is the most abundant molecule in the oxygen-rich material, the rate of gas cooling due to $\mathrm{H}_{2}$ (Hartquist et al. 1980) is much smaller than that due to the dustgas collisions.

Next to $\mathrm{H}_{2}$ the most abundant molecule in oxygen-rich matter is probably the $\mathrm{H}_{2} \mathrm{O}$ molecule (Goldreich \& Scoville 1976; Groenewegen 1994). Neufeld et al. (1995) obtain cooling functions for five molecules and two atomic species that are potentially important coolants $\left(\mathrm{H}_{2}, \mathrm{H}_{2} \mathrm{O}, \mathrm{CO}, \mathrm{O}_{2}, \mathrm{HCl}, \mathrm{C}\right.$, and $\left.\mathrm{O}\right)$, and show that, for a temperature of about $200 \mathrm{~K}$ and hydrogen concentration $10^{6} \lesssim N_{\mathrm{H}_{2}} \lesssim 10^{9} \mathrm{~cm}^{-3}$, the gas cools mostly by $\mathrm{H}_{2} \mathrm{O}$ molecules. The heat loss rate of the gas due to radiative cooling by $\mathrm{H}_{2} \mathrm{O}$ is

$q_{\mathrm{H}_{2} \mathrm{O}}=\sum N_{\mathrm{H}_{2} \mathrm{O}} h v_{u l}\left(n_{l} C_{l u}-n_{u} C_{u l}\right)$,

where the sum is over all collisional transitions in the water molecule, $C_{l u}$ and $C_{u l}$ are the rates of the collisional excitation and de-excitation. In principle, the value of $q_{\mathrm{H}_{2} \mathrm{O}}$ can be negative, which means that the radiative cooling in water lines is less than the heating due to absorption of dust radiation.

In order to test our code, we compute the cooling rate due to water lines. Figure 3 shows the dependence of $q_{\mathrm{H}_{2} \mathrm{O}} /\left(N_{\mathrm{H}_{2}} N_{\mathrm{H}_{2} \mathrm{O}}\right)$ on $N_{\mathrm{H}_{2}} N_{\mathrm{H}_{2} \mathrm{O}} H$. First, we take into account only the ground vibrational state in the water molecule and then include the firstexcited vibrational level. Our results are similar to those obtained by Neufeld \& Melnick (1987), who accounted for the first 179 rotational levels of the ground vibrational state. The small difference can be explained by the different number of rotational levels included. In the presence of dust with $T_{\mathrm{d}}=T$ the cooling is reduced because dust grains trap part of the radiation from water molecules in the medium. If one includes the firstexited vibrational state in the water molecule, the cooling rate becomes larger because of additional vibration-rotational collisional transitions and rotational transitions in the first-exited vibrational state, but with dust there is no difference.

A rather general form of the thermal balance equation for the gas is

$q_{\text {dust }}=q_{\mathrm{H}_{2} \mathrm{O}}$.

The thermal balance for the mixture of the gas and different dust types (noted by index $i$ ) is

$\sum N_{\mathrm{d}, i} A_{\mathrm{d}, i} N_{\mathrm{H}_{2}} V_{t} k\left(T_{\mathrm{d}, i}-T\right)=q_{\mathrm{H}_{2} \mathrm{O}}$.

The gas temperature can be obtained by simultaneously solving this equation with the population balance equation for a water molecule (see Sect. 2.3). Such a system of equations is non-linear and is solved by iterations. If the radiative cooling by $\mathrm{H}_{2} \mathrm{O}$ is small, the gas temperature is expressed by a simple relation

$T=\frac{\sum_{i} N_{\mathrm{d}, i} A_{\mathrm{d}, i} T_{\mathrm{d}, i}}{\sum_{i} N_{\mathrm{d}, i} A_{\mathrm{d}, i}}=\frac{\sum_{i} f_{\mathrm{d}, i} T_{\mathrm{d}, i} / \bar{\rho}_{i} a_{i}}{\sum_{i} f_{\mathrm{d}, i} / \bar{\rho}_{i} a_{i}}$

In the case of the grain size distribution, the energy balance for the gas is

$\frac{3 m_{\mathrm{p}}}{2 \bar{\rho}} N_{\mathrm{H}_{2}}^{2} V_{t} k \int_{a_{\min }}^{a_{\max }}\left[T_{\mathrm{d}}(a)-T\right] a^{-1} \mathrm{~d} f_{\mathrm{d}}=q_{\mathrm{H}_{2} \mathrm{O}}$.

When various dust types are present, some can cool the gas while others can heat it.

We illustrate the effect of water cooling with a simple example of two dust types mixed with hydrogen and water. We consider equal mass fractions of circumstellar silicate and amorphous carbon with total $f_{\mathrm{d}}=10^{-2}$. Silicate is cooler, while carbon is hotter. It is clear from Eq. (30) that small grains are more effective in heating (and cooling) the gas. Figure 4 compares the water cooling rate to that due to collisions with silicates for different grain sizes $a=0.01,0.1$, and $1 \mu \mathrm{m}$ as a function of the dilution factor. We see that the radiative cooling rate due to $\mathrm{H}_{2} \mathrm{O}$ is about two orders of magnitude smaller than the cooling due to collisions with $0.01 \mu \mathrm{m}$ grains, while the efficiency of these two mechanisms is comparable for $1 \mu \mathrm{m}$ grains.

The effect of radiative cooling by $\mathrm{H}_{2} \mathrm{O}$ on the gas temperature does not depend only on the size of the dust grains, but also on the hydrogen concentration. This is because the heating and cooling rates have different dependencies on $N_{\mathrm{H}_{2}}: q_{\text {dust }}\left(N_{\mathrm{H}_{2}}\right) \propto$ $N_{\mathrm{H}_{2}}^{2} f_{\mathrm{d}}$ (see Eqs. (23), (30)) and $q_{\mathrm{H}_{2} \mathrm{O}}\left(N_{\mathrm{H}_{2}}\right) \propto\left(N_{\mathrm{H}_{2}} N_{\mathrm{H}_{2} \mathrm{O}}\right)^{1 / 2} \propto$ $N_{\mathrm{H}_{2}} f_{\mathrm{H}_{2} \mathrm{O}}^{1 / 2}$ (see Fig. 3). In Fig. 5 we illustrate the dependence of $T$ on $N_{\mathrm{H}_{2}}$ for the dilution factor $W=5 \times 10^{-6}$ (where water cooling is most effective, see Fig. 4). At high $N_{\mathrm{H}_{2}}$ the gas temperature takes an intermediate value given by Eq. (29). In the case of $0.01 \mu \mathrm{m}$ grains, $\mathrm{H}_{2} \mathrm{O}$ radiative cooling is negligible for $N_{\mathrm{H}_{2}} \gtrsim 10^{8} \mathrm{~cm}^{-3}$, while in the case of 0.1 and $1 \mu \mathrm{m}$ grains, water cooling is important even for $N_{\mathrm{H}_{2}} \lesssim 10^{9} \mathrm{~cm}^{-3}$. Moreover, for large grains and $N_{\mathrm{H}_{2}} \lesssim 10^{7} \mathrm{~cm}^{-3}$ water cooling is so effective that the gas becomes cooler than the cold dust (silicate). 


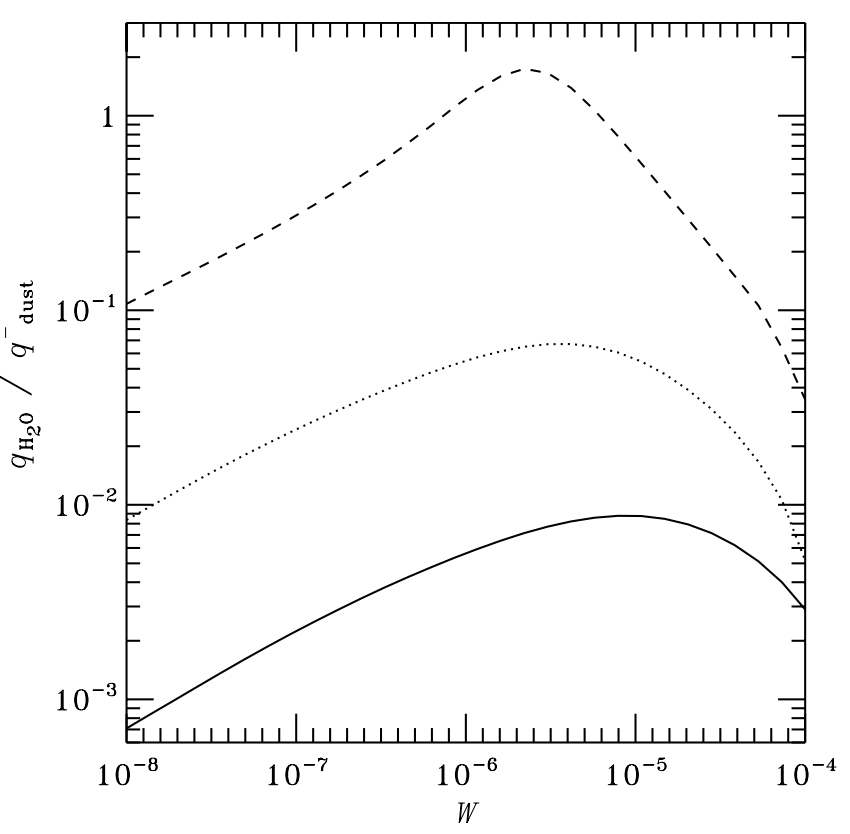

Fig. 4. Dependence of the ratio $q_{\mathrm{H}_{2} \mathrm{O}} / q_{\text {dust }}^{-}$on dilution factor $W$ for a circumstellar silicate and amorphous carbon dust mixture at the fixed values of slab half-thickness $H=10^{14} \mathrm{~cm}$, the gas concentration $N_{\mathrm{H}_{2}}=10^{8} \mathrm{~cm}^{-3}$, water mass fraction $f_{\mathrm{H}_{2} \mathrm{O}}=10^{-4}$, dust mass fraction $f_{\mathrm{d}}=10^{-2}$, temperature of the star $T_{*}=3000 \mathrm{~K}$ and different grain size $a=0.01 \mu \mathrm{m}$ (solid curve), $0.1 \mu \mathrm{m}$ (dotted curve), $1 \mu \mathrm{m}$ (dashed curve), assuming the same mass fraction of the two dust types.

In the winds from late-type stars, the radiation pressure from a star accelerates the dust which can drift through the gas, providing an additional source of gas heating (see, for example, Goldreich \& Scoville 1976)

$q_{\mathrm{drift}} \simeq \frac{1}{2} N_{\mathrm{H}_{2}} 2 m_{\mathrm{p}} \sum_{i} N_{\mathrm{d}, i} A_{\mathrm{d}, i} V_{\mathrm{d}, i}^{3}$,

where $V_{\mathrm{d}}$ is the drift velocity. This term should be added to the balance Eqs. (27) and (28).

\section{Results}

\section{1. de Jong model}

Let us first discuss how the $6_{16}-5_{23}$ water maser works if no dust is present in the medium. We will thus have a benchmark to which we can compare our dusty models. De Jong (1973) considered a gas cloud consisting of a mixture of water vapor and hydrogen. He showed that upon approach to the surface of the cloud the optical depth in the lines decreases and the thermal equilibrium breaks down. This occurs at different depths in different lines and over some range of depths some levels become relatively overpopulated, leading to population inversion in some transitions. The collisional rates of the water molecule with hydrogen were not known at that time, and he used approximate expressions. We repeat these calculations using modern collisional rates from Green et al. (1993). To find the main transitions participating in the maser pumping mechanism we compared the population fluxes $F_{u l}=n_{u} W_{u l}-n_{l} W_{l u}$ (where $W_{u l}$ and $W_{l u}$ are total transition probabilities) for all

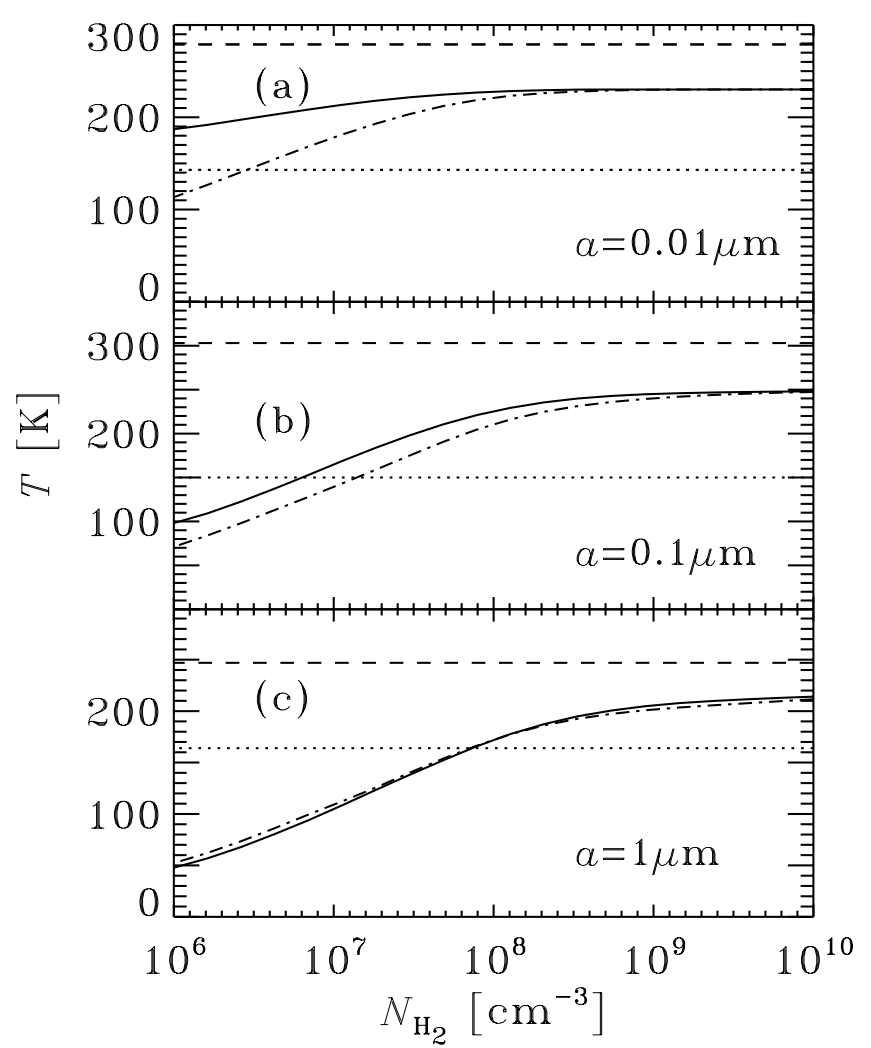

Fig. 5. Gas temperature as a function of hydrogen concentration in a slab of half-thickness $H=10^{14} \mathrm{~cm}$ in the radiation field with $W=$ $5 \times 10^{-6}$ and $T_{*}=3000 \mathrm{~K}$ and different grain sizes a) $a=0.01 \mu \mathrm{m}$, b) $0.1 \mu \mathrm{m}$, c) $1 \mu \mathrm{m}$. Water fraction of $f_{\mathrm{H}_{2} \mathrm{O}}=10^{-4}$ (solid curves) and $f_{\mathrm{H}_{2} \mathrm{O}}=10^{-3}$ (dot-dashed curves) is assumed. Dust of two types, silicate and amorphous carbon, is present with equal mass fraction and total $f_{\mathrm{d}}=10^{-2}$. Dust temperatures of the silicate and amorphous carbon are shown by dotted and dashed lines, respectively.

possible pure rotational and ro-vibrational transitions of ortho$\mathrm{H}_{2} \mathrm{O}$ molecule in the ground and first-excited vibrational state.

We find that for $N_{\mathrm{H}_{2}} \gtrsim 10^{8} \mathrm{~cm}^{-3}$ and $f_{\mathrm{H}_{2} \mathrm{O}} \gtrsim 10^{-5}$ the main cycle of the maser pumping is $5_{23} \stackrel{161 \mu \mathrm{m}}{\longrightarrow} 5_{32} \stackrel{99 \mu \mathrm{m}}{\longrightarrow} 5_{41} \stackrel{61 \mu \mathrm{m}}{\longrightarrow} 6_{16}$ (solid lines in Fig. 6). The upward transitions $5_{23} \rightarrow 5_{32}$ and $5_{32} \rightarrow 5_{41}$ are dominated by collisions and therefore, water molecules are collisionally excited from $5_{23}$ to $5_{41}$ level through $5_{32}$ level. Radiative de-excitation from $5_{41}$ to $6_{16}$ produces the heat sink in the maser pumping cycle, when radiative rate of this transition is larger than the collisional one and the optical depth in the corresponding line is small. In the remaining range of parameters $N_{\mathrm{H}_{2}}$ and $f_{\mathrm{H}_{2} \mathrm{O}}$, the main cycle of the maser pumping is $5_{23} \stackrel{45 \mu \mathrm{m}}{\longrightarrow} 4_{14} \stackrel{28 \mu \mathrm{m}}{\longrightarrow} 7_{07} \stackrel{72 \mu \mathrm{m}}{\longrightarrow} 6_{16}$. Water molecules are radiatively de-excited from the $5_{23}$ to $4_{14}$ level, collisionally excited to the $7_{07}$ level, and again radiatively deexcited to $6_{16}$ closing the pumping cycle. The pumping cycles found by us appear to be different from those considered by de Jong, probably because of more accurate collisional rates in our calculations.

In Fig. 7 (case 1, dashed curves) we present the maser absorption coefficient as a function of the slab half-thickness. The maser disappears at $H \gtrsim 10^{15} \mathrm{~cm}$, if $N_{\mathrm{H}_{2}}=10^{8} \mathrm{~cm}^{-3}$, when the probability of photon escape in $5_{41} \rightarrow 6_{16}$ transition 


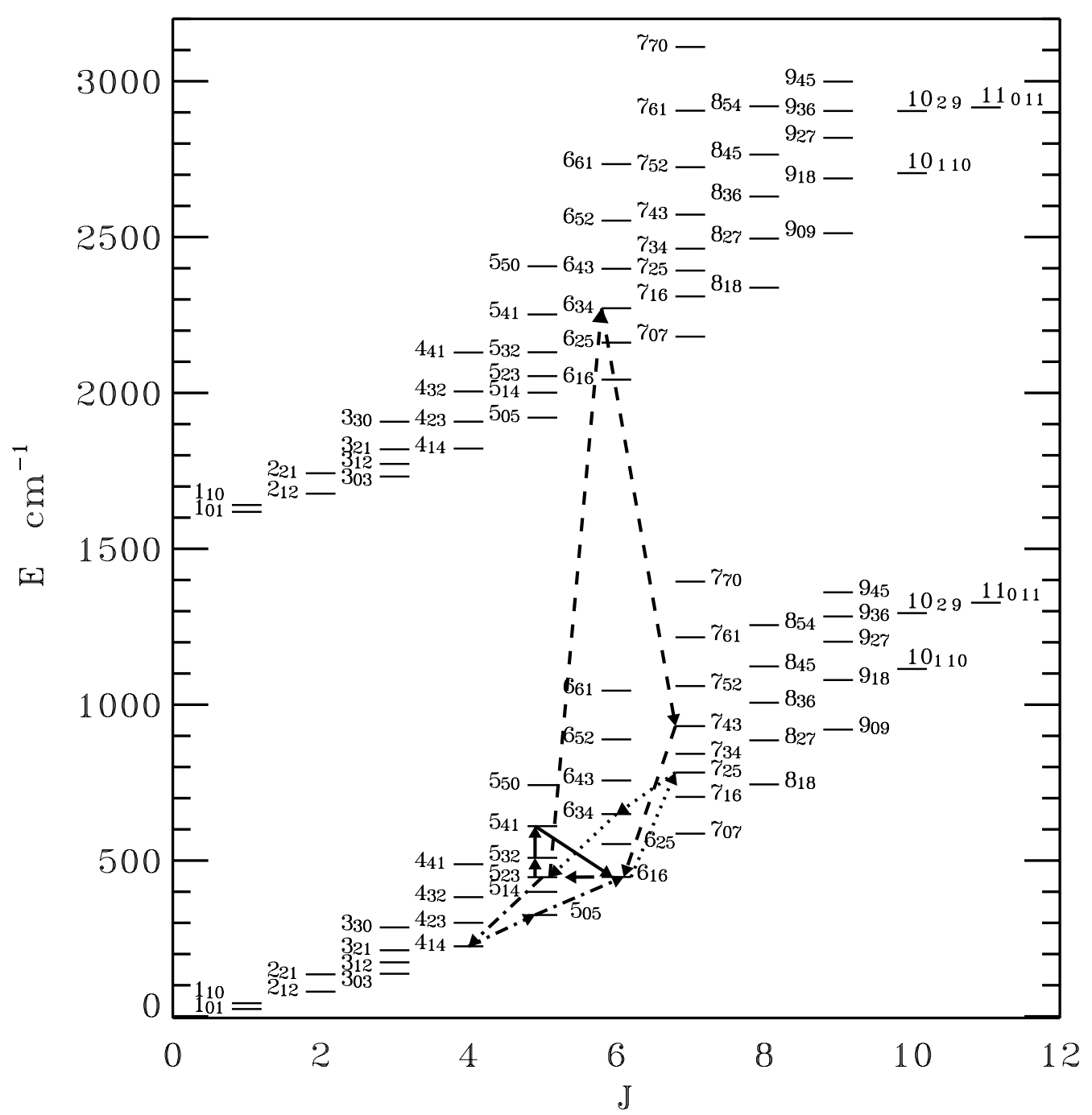

Fig. 6. System of the rotational energy levels of ortho- $\mathrm{H}_{2} \mathrm{O}$ molecule in the ground and first-excited (010) vibrational state. Arrows show the main cycles of the maser pumping in the following cases: Deguchi model (dot-dashed lines), de Jong model (solid lines), two-dust model (dashed lines). The cycle extinguishing the maser effect at large $H$ for the combinations of the ice with any other dust is shown by dotted lines.

(heat sink) becomes comparable to the rate of collisions, i.e. $C_{5_{41} \rightarrow 6_{16}} \simeq p A_{5_{41} \rightarrow 6_{16}}$ (see Eqs. (1), (10)) and BP04). For the same water concentration but higher hydrogen density of $N_{\mathrm{H}_{2}}=10^{9} \mathrm{~cm}^{-3}$, this condition is satisfied at smaller line optical depth $\tau$ corresponding to $H \sim 10^{14} \mathrm{~cm}$, where the maser disappears.

\subsection{Influence of dust}

Let us now investigate the influence of the dust on the inversion of the maser level populations. If dust (we consider $0.01 \mu \mathrm{m}$ circumstellar silicate grains) is of the same temperature as the gas, the maser efficiency drops at large depths (compare case 2, dotted curves in Fig. 7 to case 1, dashed curves). This happens because dust traps radiation from water molecules in the medium, while the de Jong mechanism is based on the escape of photons from the surface.

We have not specified any mechanism of gas heating. In the models considered above we have assumed certain gas and dust temperatures without considering energy balance. If there is no relative motion between the dust and the gas, the only efficient mechanism of gas heating is the energy exchange with the dust. If only one type of dust is present, the gas temperature can be at most that of the dust, and if water cooling is important then it is much smaller. The hotter dust not only heats the gas, but strongly affects the maser operation because of the influences of the dust radiation.

Goldreich \& Kwan (1974) proposed that the inversion of the maser level populations $6_{16}-5_{23}$ can arise due to the excitation of the first-excited vibration state (010) of the ortho$\mathrm{H}_{2} \mathrm{O}$ molecule by $6.3 \mu \mathrm{m}$ dust radiation, while the heat sink is realized through collisions with cooler hydrogen. This model has been criticized by Deguchi (1981), who has pointed out that at high concentrations needed for the collisional sink, the levels at the ground vibrational state will be thermalized.

To check this scenario, we considered amorphous carbon grains of size $a=1 \mu \mathrm{m}$ in the radiation field with stellar temperature $T_{*}=3000 \mathrm{~K}$ and dilution factor $W=5 \times 10^{-6}$. The dust temperature $T_{\mathrm{d}}=279 \mathrm{~K}$ is determined from the thermal balance Eq. (24). The gas temperature, which is computed 


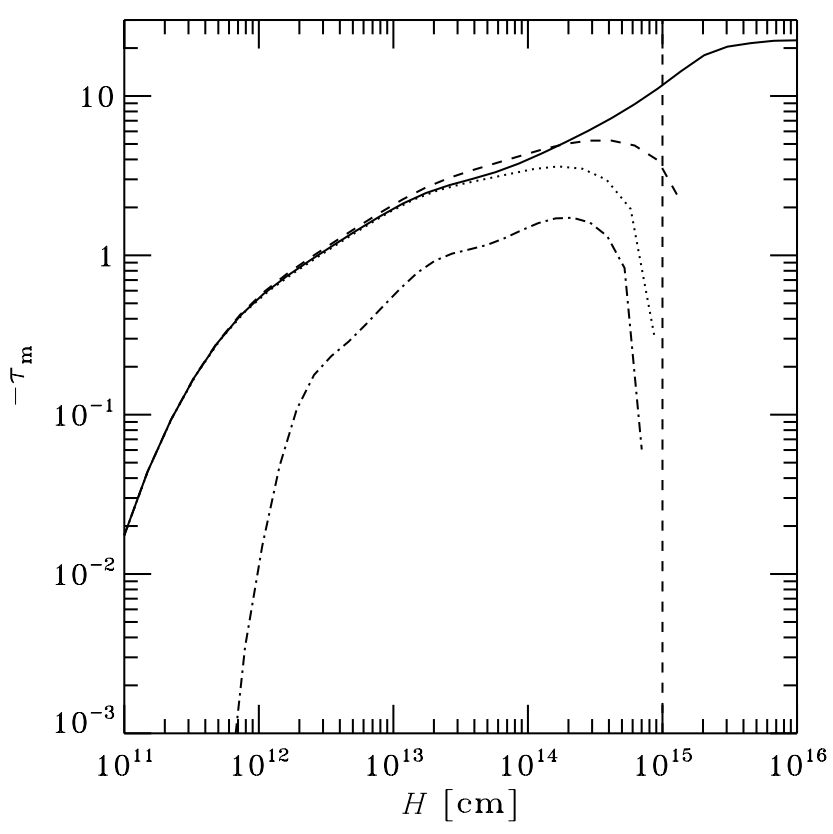

Fig. 7. The optical depth in the $6_{16}-5_{23}$ maser line as a function of the half-thickness of the slab for $N_{\mathrm{H}_{2}}=10^{8} \mathrm{~cm}^{-3}, f_{\mathrm{H}_{2} \mathrm{O}}=10^{-3}$. The following cases are considered: (1) de Jong mechanism in the water-hydrogen mixture (without dust) of $T=230 \mathrm{~K}$ (dashed curves); (2) same as previous, but with circumstellar silicate grains of $a=0.01$ $\mu \mathrm{m}$ and $T_{\mathrm{d}}=T$ (dotted curves); (3) gas mixture with amorphous carbon grains of $a=1 \mu \mathrm{m}$ and $T_{\mathrm{d}}=279 \mathrm{~K}$. The gas temperature is calculated self-consistently, using thermal balance Eq. (28) (dotdashed curves); (4) circumstellar silicate grains of $a=0.01 \mu \mathrm{m}$ and $T_{\mathrm{d}}^{\mathrm{c}}=143 \mathrm{~K}$, gas with $T=230 \mathrm{~K}$, and $0.01 \mu \mathrm{m}$ amorphous carbon grains with $T_{\mathrm{d}}^{\mathrm{h}}=279 \mathrm{~K}$ (solid curves). Dust temperatures correspond to the dilution factor $W=5 \times 10^{-6}$ and stellar temperature $T_{*}=3000 \mathrm{~K}$. We assume $f_{\mathrm{d}}^{\mathrm{h}}=f_{\mathrm{d}}^{\mathrm{c}}=f_{\mathrm{d}} / 2, f_{\mathrm{d}}=10^{-2}$, and $\mu_{\min }=1$. On the right hand side of the vertical dashed lines the dust is optically thick.

self-consistently from Eq. (28) accounting for the water cooling and the heating by collisions with the dust, varies from $43 \mathrm{~K}$ at $H=10^{11} \mathrm{~cm}$ to $276 \mathrm{~K}$ at $H=10^{16} \mathrm{~cm}\left(\right.$ for $\left.N_{\mathrm{H}_{2}}=10^{8} \mathrm{~cm}^{-3}\right)$. The inversion of maser levels (see case 3 , dot-dashed curves in Fig. 7) is smaller than in previously considered cases and is still produced by the de Jong mechanism. At small $H$ the radiative cooling by water is so intense that the gas temperature becomes too low for maser pumping. In spite of inability of the hot dust to efficiently pump the maser, the presence of the hot dust settles the problem of the gas heating.

Of course, if there are additional mechanisms of gas heating (e.g. due to dynamic friction with rapidly moving dust, see Eq. (31)), the gas temperature can exceed the dust temperature by a large factor. In this situation the maser is very effective (as was discussed for example by Chandra et al. 1984a; Collison \& Watson 1995; Yates et al. 1997; BP04).

\subsection{Hot and cold dust pumping mechanism}

The late-type stars often show emission of different types of dust (Danchi et al. 1994). Thus, it is natural to assume that two dust types are present simultaneously. Since dust temperatures

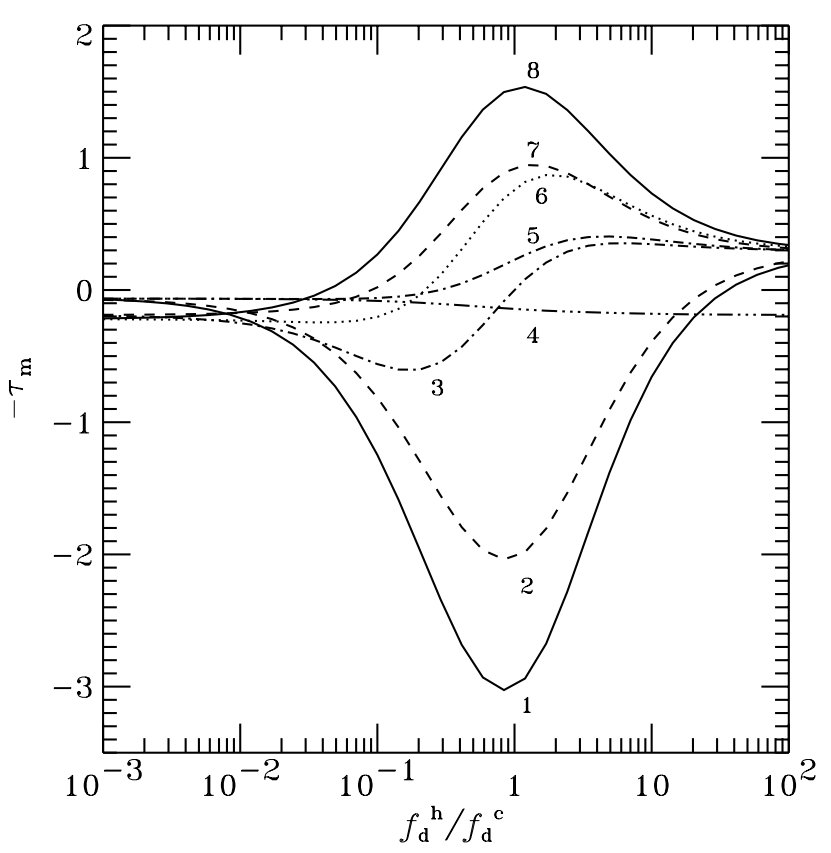

Fig. 8. The optical depth $\tau_{\mathrm{m}}$ in the $6_{16} \rightarrow 5_{23}$ transition as a function of the ratio $f_{\mathrm{d}}^{\mathrm{h}} / f_{\mathrm{d}}^{\mathrm{c}}$ for different cold - hot dust combinations: amorphous ice and graphite (1), crystalline ice and graphite (2), crystalline ice and amorphous carbon (3), crystalline ice and circumstellar silicate (4), circumstellar silicate and graphite (5), astronomical silicate and graphite (6), circumstellar silicate and amorphous carbon (7), astronomical silicate and amorphous carbon (8). The parameters are $H=10^{14} \mathrm{~cm}, W=5 \times 10^{-6}, T_{*}=3000 \mathrm{~K}$ and the grain size $a=0.01 \mu \mathrm{m}$.

differ (see Fig. 2), collisions with one dust can heat the gas, while with another can cool it. The gas temperature then takes an intermediate value (see Fig. 5).

What pairs of dust produce the largest inversion? From Fig. 2 we see that a significant temperature difference can be achieved for dust types where one is graphite or amorphous carbon and another one is any of the remaining (silicates or ice). For completeness, we also consider a mixture of crystalline ice and circumstellar silicate, in spite of the fact that the temperature difference is not so large. Figure 8 shows the dependence of the optical depth in the maser line $\tau_{\mathrm{m}}$ on the ratio of the hotto-cold mass fractions $f_{\mathrm{d}}^{\mathrm{h}} / f_{\mathrm{d}}^{\mathrm{c}}$ for different dust combinations. We see that the strongest maser (i.e. largest negative optical depth $\tau_{\mathrm{m}}$ ) occurs at a ratio $f_{\mathrm{d}}^{\mathrm{h}} / f_{\mathrm{d}}^{\mathrm{c}} \simeq 1$ for combinations involving graphite (or amorphous carbon) and silicates. We further assume the same mass fraction of the cold and hot dust, $f_{\mathrm{d}}^{\mathrm{h}}=f_{\mathrm{d}}^{\mathrm{c}}$.

Let us now consider a mixture of $0.01 \mu \mathrm{m}$ circumstellar silicate and amorphous carbon grains. Their temperatures, in the radiation field with $W=5 \times 10^{-6}$ and $T_{*}=3000 \mathrm{~K}$, are $T_{\mathrm{d}}^{\mathrm{cs}}=143 \mathrm{~K}$ and $T_{\mathrm{d}}^{\mathrm{ac}}=279 \mathrm{~K}$, respectively. The gas temperature of $T=230 \mathrm{~K}$ is fully determined by the collisions with dust which are more efficient than water radiative cooling for small grains (see Sect. 2.6). The resulting maser strength as a function of the slab thickness is shown in Fig. 7 (case 4, solid curves). We see that at $H \lesssim 10^{14} \mathrm{~cm}$, the inversion is identical to that in the dust-free case, implying operation of the de Jong 


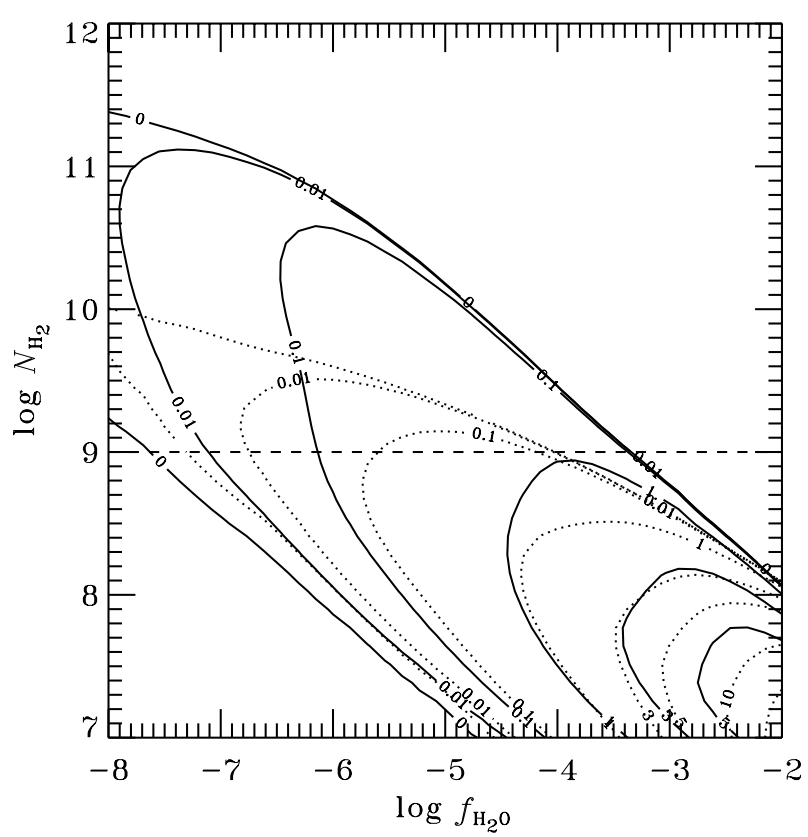

Fig. 9. Contour plots of levels of constant the $6_{16} \rightarrow 5_{23}$ maser optical depth $-\tau_{\mathrm{m}}$. Solid curves correspond to the case of the circumstellar silicate dust with $T_{\mathrm{d}}^{\mathrm{c}}=143 \mathrm{~K}$ and amorphous carbon with $T_{\mathrm{d}}^{\mathrm{h}}=279 \mathrm{~K}$; dotted curves correspond to the case of equal gas and dust temperatures $T=T_{\mathrm{d}}=230 \mathrm{~K}$. The size of the dust grains is $a=0.01 \mu \mathrm{m}$ and the slab half thickness is $H=10^{14} \mathrm{~cm}$, the mass fraction of the silicate and carbon dust is the same $f_{\mathrm{d}}^{\mathrm{c}}=f_{\mathrm{d}}^{\mathrm{h}}=5 \times 10^{-3}$. On the upper side of the horizontal dashed lines the dust is optically thick.

mechanism. At larger $H$, the maser absorption coefficient is almost constant resulting in a linear increase of the maser optical depth. At even higher $H \gtrsim 3 \times 10^{15} \mathrm{~cm}$ the maser saturates (we used $\mu_{\min }=1$ in these calculations, i.e. almost spherical maser, see Eq. (8)). Thus, we see that the presence of two dust types is rather efficient in heating the gas as well as keeping a large inversion deep in the cloud. For $N_{\mathrm{H}_{2}} H \gtrsim 10^{23} \mathrm{~cm}^{-2}$ the medium becomes optically thick to absorption by dust, and a large difference between the gas and dust temperatures is unlikely to be produced in real astrophysical environments (unless there is additional heating, of course).

In Fig. 9 we present contour plots of levels of the constant $6_{16} \rightarrow 5_{23}$ maser optical depth $\tau_{\mathrm{m}}$ at the plane hydrogen concentration - water-to-gas mass ratio. The gas temperature is calculated self-consistently from the thermal balance Eq. (28). For large $N_{\mathrm{H}_{2}}$ or small water content, the gas temperature is $230 \mathrm{~K}$, while for smaller $N_{\mathrm{H}_{2}}$ and large $f_{\mathrm{H}_{2} \mathrm{O}}$, the gas cooling by $\mathrm{H}_{2} \mathrm{O}$ is important and $T$ decreases down to $150 \mathrm{~K}$ (lower right corner in Fig. 9). We assume that the dust temperatures are constant in the whole range of $N_{\mathrm{H}_{2}}$. However, for $N_{\mathrm{H}_{2}} \gtrsim 10^{9} \mathrm{~cm}^{-3}$ (and our assumed $H=10^{14} \mathrm{~cm}$ ) the medium becomes optically thick to the dust absorption and therefore the gas and dust temperatures equalize. To investigate the maser effect in the case of equal gas and dust temperatures, we also present in Fig. 9 (dotted curves) the maser optical depth for $T=T_{\mathrm{d}}=230 \mathrm{~K}$. In that case the inversion of maser levels exists up to $N_{\mathrm{H}_{2}} \lesssim 10^{10} \mathrm{~cm}^{-3}$ and the maser is weak in the range $10^{9} \lesssim N_{\mathrm{H}_{2}} \lesssim 10^{10} \mathrm{~cm}^{-3}$. Thus, the $6_{16} \rightarrow 5_{23}$ maser is mostly effective in the medium with $\tau_{\mathrm{d}} \lesssim 1$ (see Eq. (20)).

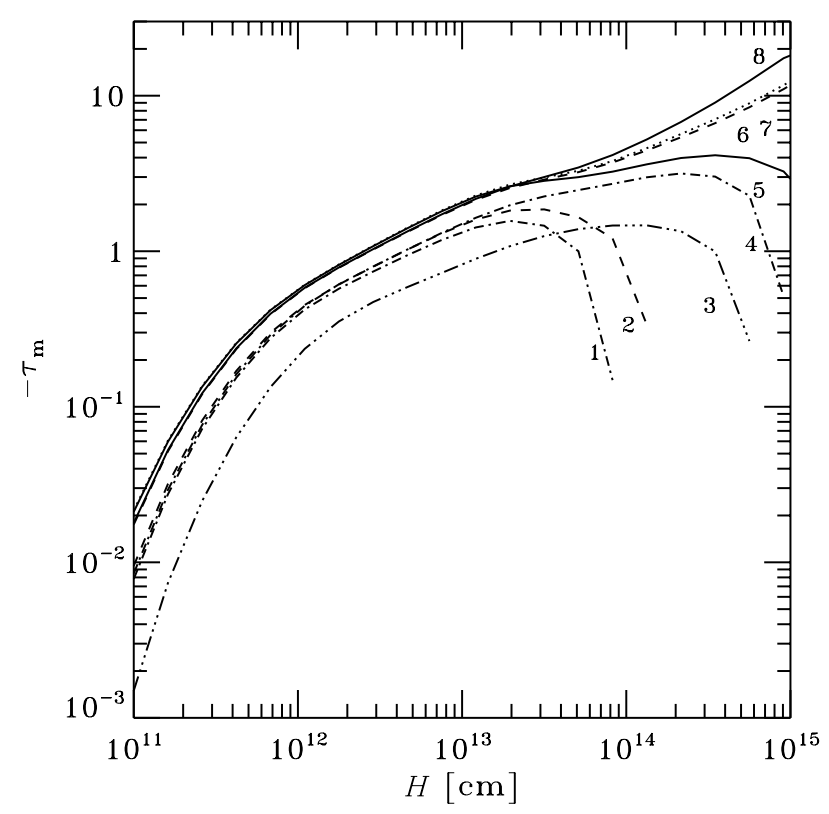

Fig. 10. The optical depth in the $6_{16}-5_{23}$ maser line as a function of the slab half-thickness for the same cold-hot dust combination as in Fig. 8 and the following set of parameters: $a=0.01 \mu \mathrm{m}, T_{*}=3000 \mathrm{~K}$, $W=5 \times 10^{-6}, f_{\mathrm{d}}^{\mathrm{h}}=f_{\mathrm{d}}^{\mathrm{c}}=5 \times 10^{-3}$.

The $6_{16}-5_{23}$ maser optical depth as a function of $H$ is shown in Fig. 10 for the same cold-hot dust combinations as in Fig. 8. We see that the maser is less effective for the combinations of ice with other dust types and most effective for the combinations of silicates with amorphous carbon. The maser works due to the de Jong mechanism when $H \lesssim 10^{14} \mathrm{~cm}$, while for the silicates-carbon (graphite) mixtures the inversion exists also at larger $H$, where the maser action is controlled by the dust.

The analysis of the population fluxes $F_{u l}$ reveals that, unlike the cold dust - hot gas mechanism operating on the pumping cycle $5_{23} \stackrel{45 \mu \mathrm{m}}{\longrightarrow} 4_{14} \stackrel{100 \mu \mathrm{m}}{\longrightarrow} 5_{05} \stackrel{82 \mu \mathrm{m}}{\longrightarrow} 6_{16} \quad$ (Deguchi 1981; BP04; see dot-dashed lines in Fig. 6), the main pumping cycle of the two-dust mechanism is $5_{23} \stackrel{5.5 \mu \mathrm{m}}{\longrightarrow} 6_{34}^{\mathrm{E}} \stackrel{7.6 \mu \mathrm{m}}{\longrightarrow} 7_{43} \stackrel{21 \mu \mathrm{m}}{\longrightarrow} 6_{16}$ (dashed lines in Fig. 6). In other words, the presence of the hot dust extinguishes maser pumping based on the absorption of $45 \mu \mathrm{m}$ photons, because the absorption coefficients of silicates and amorphous carbon near $45 \mu \mathrm{m}$ are similar (see Fig. 1).

Since the absorption coefficient of the amorphous carbon at $5.5 \mu \mathrm{m}$ is much larger than that of silicates, the $5_{23} \stackrel{5.5 \mu \mathrm{m}}{\longrightarrow} 6_{34}^{\mathrm{E}}$ transition is dominated by the hot dust. On the other hand, there is a peak in the absorption coefficient of the silicate dust near $20 \mu \mathrm{m}$ (see Fig. 1) and, therefore, the $743 \stackrel{21 \mu \mathrm{m}}{\longrightarrow} 6_{16}$ transition is dominated by the cold dust. Thus, level $6_{34}^{\mathrm{E}}$ is populated by absorption of the $5.5 \mu \mathrm{m}$ radiation from the hot dust, while the heat sink is realized by absorbing the $21 \mu \mathrm{m}$ photons by the cold dust. Moreover, since the Einstein coefficient for the $6_{34}^{\mathrm{E}} \rightarrow 7_{43}$ transition is large $A=2.4 \mathrm{~s}^{-1}$, water molecules are radiatively de-excited from $6_{34}^{\mathrm{E}}$ to level $7_{43}$, closing the cycle of $6_{16} \rightarrow 5_{23}$ maser pumping. 


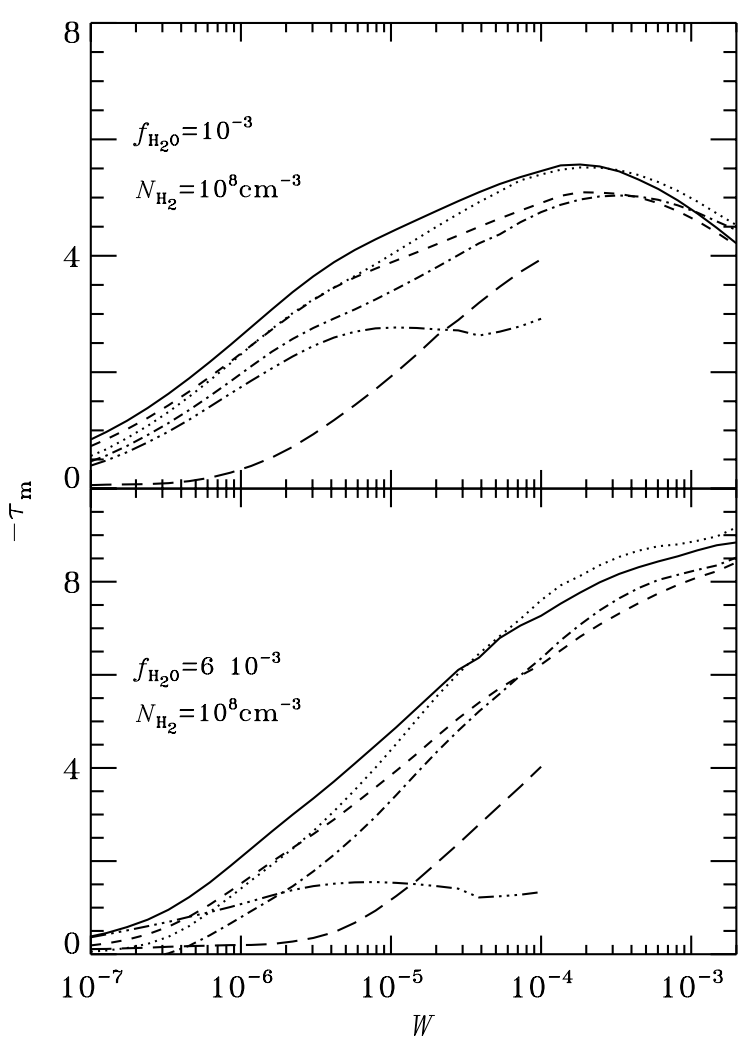

Fig. 11. The $6_{16}-5_{23}$ maser optical depth as a function of the dilution factor $W$ for different cold-hot dust combinations: astronomical silicate and amorphous carbon (solid curves), astronomical silicate and graphite (dotted curves), circumstellar silicate and amorphous carbon (dashed curves), circumstellar silicate and graphite (dot-dashed curves), crystalline ice and amorphous carbon (triple-dotdashed curves), crystalline ice and circumstellar silicate (long-dashed curves). The parameters are $f_{\mathrm{d}}^{\mathrm{h}}=f_{\mathrm{d}}^{\mathrm{c}}=5 \times 10^{-3}, a=0.01 \mu \mathrm{m}$, $H=10^{14} \mathrm{~cm}$, and $T_{*}=3000 \mathrm{~K}$. Maser efficiency for ice is not computed for $W>10^{-4}$, because of its evaporation at corresponding temperatures.

When cold ice is combined with any other hot dust the maser effect is extinguished at large $H$ because of the cycle $6_{16} \stackrel{30 \mu \mathrm{m}}{\longrightarrow} 7_{25} \stackrel{75 \mu \mathrm{m}}{\longrightarrow} 6_{34} \stackrel{49 \mu \mathrm{m}}{\longrightarrow} 5_{23}$ (dotted lines in Fig. 6). This cycle arises due to the gap in the ice absorption coefficient near $30 \mu \mathrm{m}$ (see Fig. 1). The molecules are excited from $6_{16}$ to $7_{25}$ by absorption of $30 \mu \mathrm{m}$ photons from the hot dust, which dominates this transition. De-excitation from $7_{25}$ to $5_{23}$ is realized through $7_{25} \stackrel{75 \mu \mathrm{m}}{\longrightarrow} 6_{34}$ and $6_{34} \stackrel{49 \mu \mathrm{m}}{\longrightarrow} 5_{23}$ transitions dominated by the cold ice (since its absorption coefficient is larger than that of silicates or amorphous carbon at these wavelengths) and inversion disappears.

Figure 11 shows the dependence of the maser power on the dilution factor for the dust combinations (3)-(8) considered in Figs. 8, 10. One can see that the amorphous carbon appears to be more effective than graphite in producing the inversion of the maser levels, while the astronomical silicate is more effective than the circumstellar silicate. It happens because the absorption coefficient at $21 \mu \mathrm{m}\left(7_{43} \longrightarrow 6_{16}\right.$ transition responsible for the heat sink in the maser pumping cycle) of the amorphous carbon is smaller than that of graphite, while the absorption coefficient of astronomical silicate is larger than that of circumstellar silicate (see Fig. 1). The larger the efficiency of absorption of these photons by the cold dust and the weaker the emission of these photons by the hot dust, the larger the inversion of the maser level populations.

\subsection{Grain size distribution}

In the previous section we showed that the presence of two types of dust solves the problem of gas heating as well as provides the necessary conditions for strong maser pumping. A similar effect can be achieved if there is a dust grain size distribution. In Fig. 12a we present the dependence of the dust temperature on grain size. We see that the silicate, graphite and amorphous carbon temperatures vary a lot, while the ice temperature is almost constant, because optical properties of the ice weakly depend on the grain size (see Fig. 1). The radiative cooling by water molecules strongly affects the gas temperature at small $N_{\mathrm{H}_{2}}$ where collisions with dust are less efficient (see Fig. 12b). At $N_{\mathrm{H}_{2}} \gtrsim 10^{8} \mathrm{~cm}^{-3}$ the gas temperature approaches the dust temperature of the smallest grains which dominate the gas heating (see Eq. (30)). At $N_{\mathrm{H}_{2}} \simeq 10^{7} \mathrm{~cm}^{-3}$ (for silicates at $10^{8} \mathrm{~cm}^{-3}$ ), the gas temperature becomes smaller than the minimum dust temperature.

In Fig. 13 we present the dependence of the maser optical depth as a function of the slab half-thickness. The maser absorption coefficient is maximal for $H=10^{12}-10^{13} \mathrm{~cm}$ and disappears at $H \lesssim 10^{11} \mathrm{~cm}$. It can be explained as follows. The smaller $H$, the smaller is the trapping of photons in the slab and the larger is the radiative cooling of the gas by water. At small $H$ the gas temperature thus becomes too low for maser pumping and the maser disappears. For ice, silicate and amorphous carbon the inversion exists only for $10^{11} \lesssim H \lesssim 10^{15} \mathrm{~cm}$, which means that here the maser is pumped due to the de Jong mechanism. Graphite and carbon dust have the highest temperatures and largest temperature variations. This causes the maser to operate even in the deep layers. We see also that the maser is stronger for ice dust than for the silicate, in spite of the fact that the ice temperature is smaller than of the silicates (see Fig. 12a). The reason is that the water cooling rate is larger in the presence of silicates than in the presence of ice. It happens because the ice traps radiation better than silicates, since $\mathrm{H}_{2} \mathrm{O}$ lines are mostly concentrated in the IR region, where the absorption coefficient of ice is larger than that of silicates.

The dependence of maser optical depth $\tau_{\mathrm{m}}$ on the hydrogen concentration and water-to-gas mass ratio is shown in Fig. 14 for the amorphous carbon dust distribution. The maser strength is large near $N_{\mathrm{H}_{2}} \simeq 10^{7} \div 10^{8} \mathrm{~cm}^{-3}$ and high water concentration. These results are similar to that for the case of the two-dust model (see Fig. 9), because the cycle of the maser pumping in these two models is the same.

\subsection{Applications}

\subsubsection{Silicate carbon star V778 Cyg}

Water masers are observed in some silicate carbon stars, e.g. V778 Cyg (Engels 1994; Engels \& Leinert 1994). This 

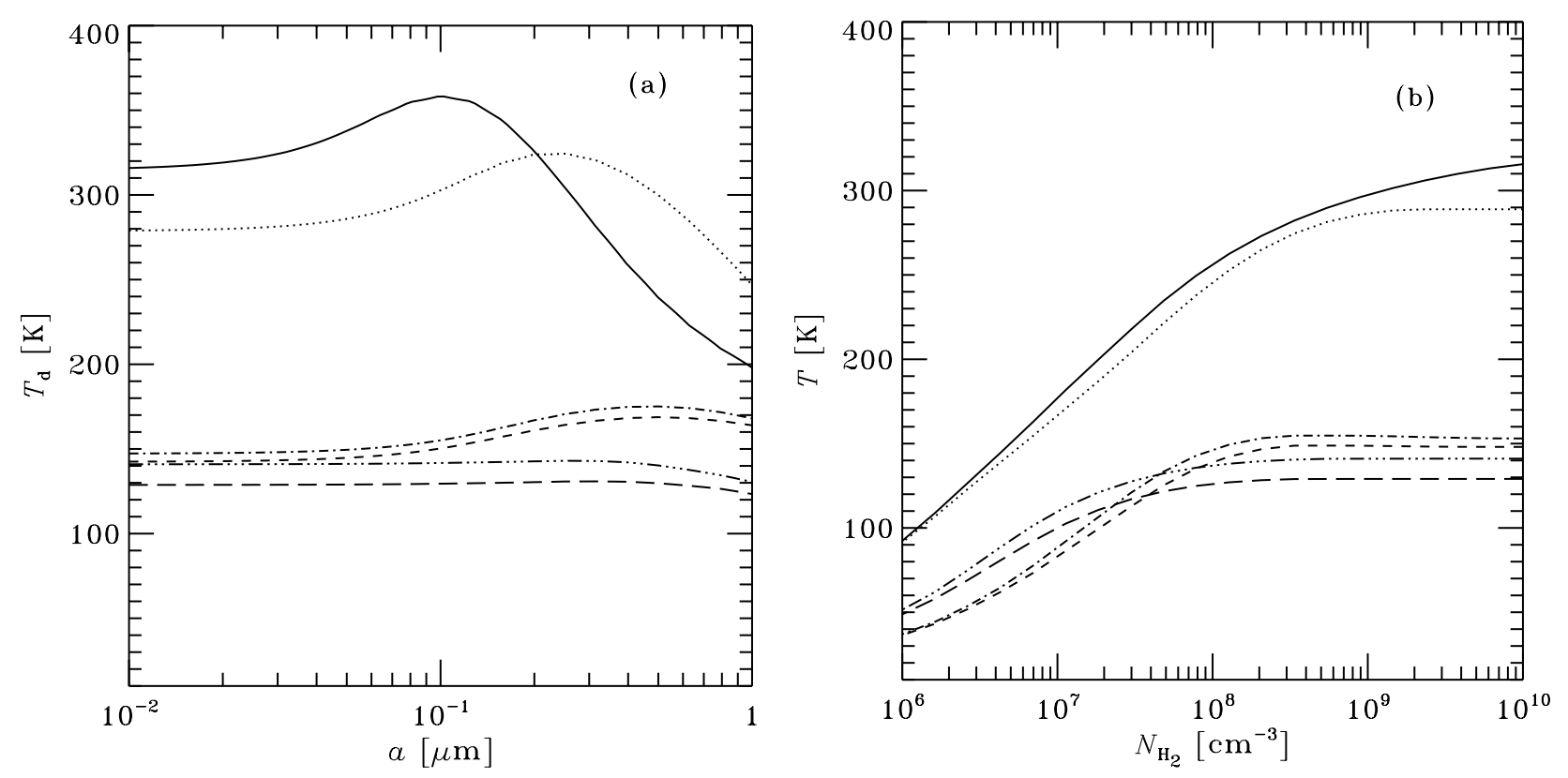

Fig. 12. a) Dependence of the dust temperature on the grain size for graphite (solid curve), amorphous carbon (dotted curve), circumstellar silicate (dashed curve), astronomical silicate (dot-dashed curve), crystalline ice (triple-dot-dashed curve), amorphous ice (long dashed curve). b) Gas temperature as a function of the hydrogen concentration for grains of the same dust types as in a) with the size distribution described by Eq. (14). Other parameters are $f_{\mathrm{H}_{2} \mathrm{O}}=10^{-3}, f_{\mathrm{d}}=10^{-2}, H=10^{14} \mathrm{~cm}, W=5 \times 10^{-6}, T_{*}=3000 \mathrm{~K}$.

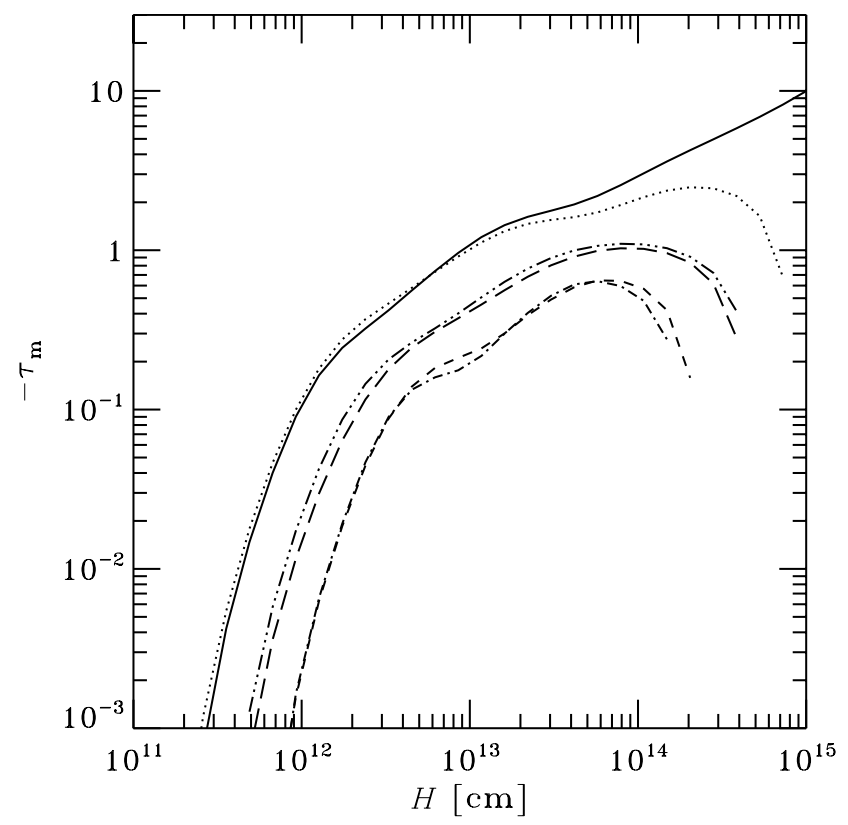

Fig. 13. The optical depth in the $6_{16}-5_{23}$ maser line as a function of the half-thickness of the slab for the same dust types as in Fig. 12 and the same parameters, $N_{\mathrm{H}_{2}}=10^{8} \mathrm{~cm}^{-3}$.

object also shows silicate dust emission features in the IR spectra (Yamamura et al. 2000). Recent detailed mapping of the masers with MERLIN revealed an elongated S-shape structure of about $32 \mathrm{AU}$ (Szczerba et al. 2005). Such a structure can be interpreted as a warped disk around a low-mass companion of the carbon star in a binary system observed almost edge-on.

The lower limit on the maser brightness temperature is $T_{\mathrm{B}}=6 \times 10^{8} \mathrm{~K}$. This requires the maser optical depth of at least

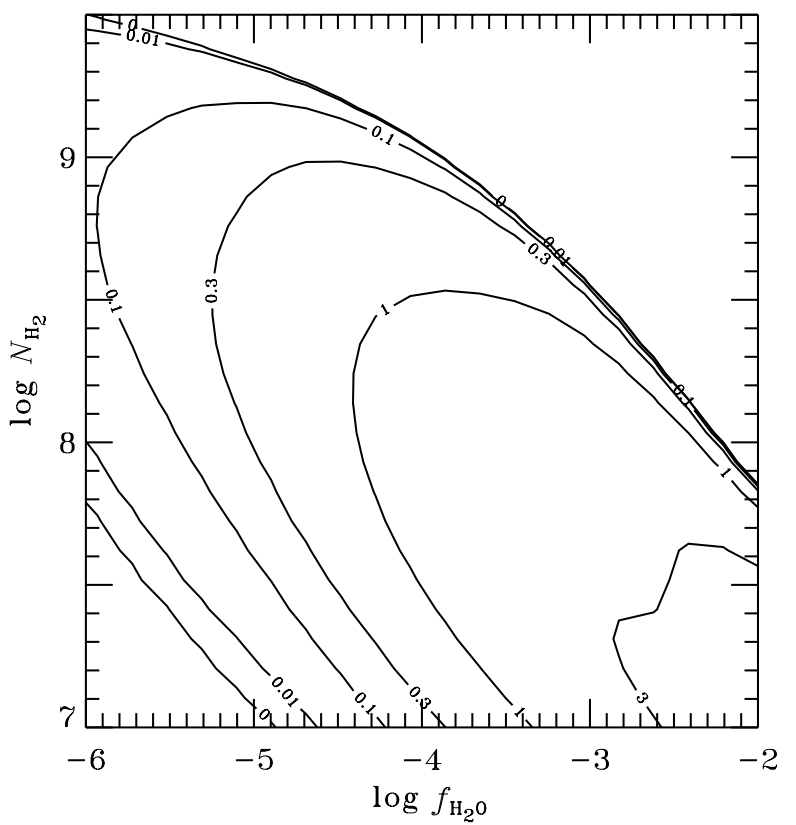

Fig. 14. Contour plots of levels of constant $6_{16} \rightarrow 5_{23}$ maser optical depth, $-\tau_{\mathrm{m}}$, for the amorphous carbon dust distribution (14). Same parameters as in Fig. 12.

$\tau=-\ln \left[T_{\mathrm{B}} / T_{\mathrm{ex}}\right] \approx-15.6$, where the excitation temperature is $\sim 100 \mathrm{~K}$. Szczerba et al. (2005) estimated the distance between the masing disk and the carbon star to be about $80 \mathrm{AU}$. With the stellar radius of $2 \mathrm{AU}$, the dilution factor is about $W=10^{-4}$.

We assume that the disk contains a mixture of $0.01 \mu \mathrm{m}$ astronomical silicate and amorphous carbon grains with the total mass fraction $f_{\mathrm{d}}=0.01$. In the stellar radiation field of a carbon star (with temperature of about $3000 \mathrm{~K}$ ), the temperature of 
the dust is $T_{\text {as }}=235 \mathrm{~K}$ and $T_{\text {ac }}=500 \mathrm{~K}$, respectively. Taking $N_{\mathrm{H}_{2}}=10^{8} \mathrm{~cm}^{-3}$ and water fraction $f_{\mathrm{H}_{2} \mathrm{O}}=6 \times 10^{-3}$ (Jeong et al. 2003), the resulting gas temperature is $T=360 \mathrm{~K}$. From Fig. 10, we get $\tau_{\mathrm{m}} \approx 8$ for $H=10^{14} \mathrm{~cm}$ which gives us the absorption coefficient $\alpha_{\mathrm{L}}^{\mathrm{m}}=\tau_{\mathrm{m}} / H=-8 \times 10^{-14} \mathrm{~cm}^{-1}$. For the projected disk radius of $R=16 \mathrm{AU}$, the coherent length in the disk is about $S=1.2 R=3 \times 10^{14} \mathrm{~cm}$. Using Eq. (9), we get $\tau=S \alpha_{\mathrm{L}}^{\mathrm{m}} / \sqrt{\pi}=-13.5$. However, for $H=5 \times 10^{13} \mathrm{~cm}(\mathrm{e} . \mathrm{g}$. away from the disk central plane), the inversion is larger by a factor 1.5 (see Fig. 11). This then gives the maximum maser optical depth of -20 which is larger than the observed lower limit. Even for a much lower water content $f_{\mathrm{H}_{2} \mathrm{O}}=10^{-3}$ we still get $\tau=-14$. Thus, collisional energy exchange with the dust can provide the gas heating which is necessary for the masers to operate.

\subsubsection{Masers from AGB winds}

Water masers from AGB stars are observed in their expanding envelopes. Four low-mass late-type stars (IKTau, U Ori, RT Vir and U Her) have been mapped by MERLIN recently (Bains et al. 2003). The data show that maser radiation comes from individual maser clouds with the apparent size of 2-4 AU (Richards et al. 1999) and the filling factor of only $\sim 0.01$. Masers are observed at typical distance from the star of 10-70 AU. The total maser photon production rate (luminosity) is about $(1-8) \times 10^{42} \mathrm{~s}^{-1}$. With the total number of clouds varying between 14 and 286 depending on a source, one can estimate a single cloud luminosity of less than $10^{41} \mathrm{~s}^{-1}$. For a spherical cloud with the radius of $\sim 2.5 \mathrm{AU}$, the maser emissivity $\Phi$ varies between $\sim 0.2$ and $\sim 0.6 \mathrm{~cm}^{-3} \mathrm{~s}^{-1}$.

Let us consider a gas-dust cloud of radius $H=4 \times 10^{13} \mathrm{~cm}$ in a radiation field of the star with $T_{*}=3000 \mathrm{~K}$ and $W=10^{-4}$ (i.e. at a distance of about $50 \mathrm{AU}$ for a $1 \mathrm{AU}$ stellar radius), and other parameters are the same as in Sect. 3.5.1. The gas temperature depends on the heating which can be provided by collisions with dust. We consider heating (a) due to the gas thermal motion (see Eq. (23)) and (b) due to the drift of grains through the gas (see Eq. (31)). If only heating due to thermal motions is considered, the gas temperature is about $360 \mathrm{~K}$ and it lies between temperatures of the cold and hot dust. The maser optical depth is $\tau_{\mathrm{m}} \approx-5$ (see dotted curve in Fig. 15). From our model we also can compute the average maser photon emissivity (see e.g. Elitzur 1991)

$\Phi=g_{u} A_{u l}\left|\Delta n_{u l}\right| N_{\mathrm{H}_{2} \mathrm{O}}\left|S_{u l}\right| K_{2}^{\mathrm{m}}$,

which is about $2 \times 10^{-3} \mathrm{~cm}^{-3} \mathrm{~s}^{-1}$, i.e. two orders of magnitudes lower than observed.

When drift heating corresponding to the velocity of only $V_{\mathrm{d}}=2 \mathrm{~km} \mathrm{~s}^{-1}$ is considered, the gas temperature becomes much larger than temperatures of the dust. The maser optical depth increases by a factor of three and the resulting power by two orders of magnitude reaching $\Phi=0.4 \mathrm{~cm}^{-3} \mathrm{~s}^{-1}$. This increase occurs because maser pumping is the most effective when gas is hotter than the dust (see, for example, BP04).

We can conclude that gas heating by collisions with dust (due to thermal motion) is not sufficient to produce observed

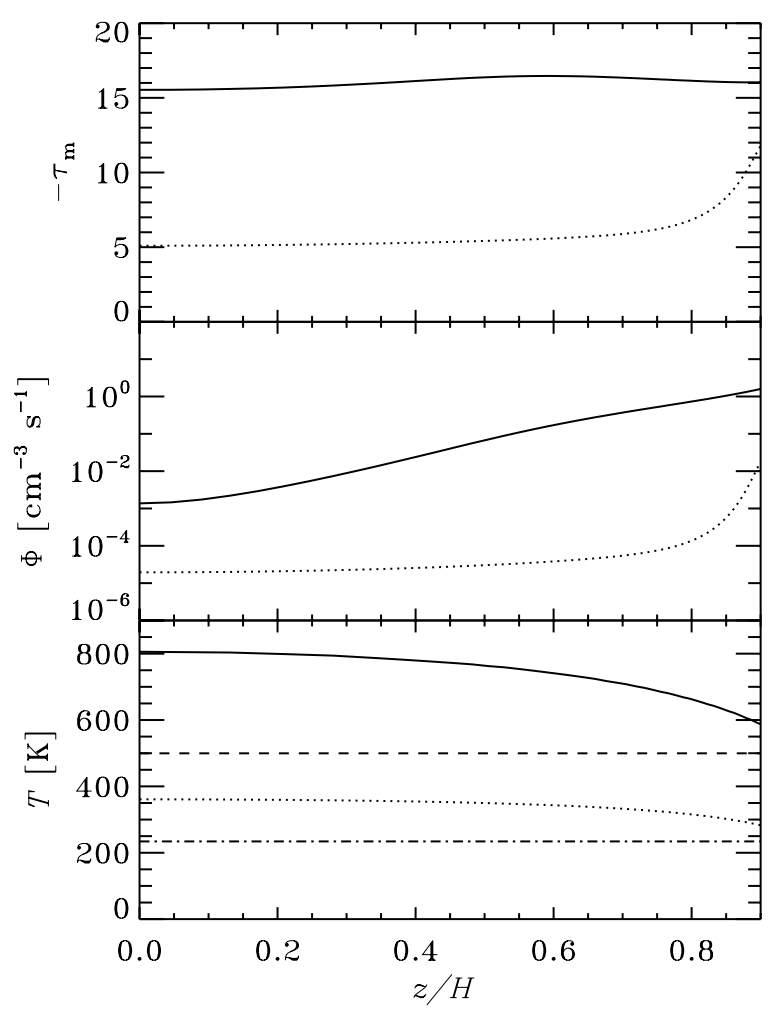

Fig. 15. Dependence of the maser optical depth, maser photon emissivity $\Phi$ [photon $\mathrm{cm}^{-3} \mathrm{~s}^{-1}$ ] and temperatures of the gas and dust on a height $z$ within a slab ( $z=0$ in the slab center). Solid curves show the case of the drift heating of the gas given by Eq. (31) and dotted curves represent the case of heating due to thermal motions (see Eq. (23)). Dashed and dot-dashed curves represent the temperatures of the astronomical silicate and amorphous carbon, respectively, calculated for $W=10^{-4}$ and $T_{*}=3000 \mathrm{~K}$. Other parameters are $N_{\mathrm{H}_{2}}=10^{8} \mathrm{~cm}^{-3}$, $f_{\mathrm{H}_{2} \mathrm{O}}=6 \times 10^{-3}, f_{\mathrm{d}}=10^{-2}, H=4 \times 10^{13} \mathrm{~cm}, a=0.01 \mu \mathrm{m}$, drift velocity $V_{\mathrm{d}}=2 \mathrm{~km} \mathrm{~s}^{-1}$.

maser luminosity, while additional drift heating by moving dust is capable of explaining the masers in AGB winds.

\section{Summary}

We have considered the maser effect in a medium consisting of a mixture of gas (hydrogen and water vapor) and dust of various types. The gas and dust temperatures and level populations of water molecule are calculated self-consistently from the system of population balance equations and thermal balance equations for the gas and dust in the radiation field of a late-type star.

When dust of different types is present, the gas interacting with the grains can be heated by one type of dust and is cooled by another. The gas temperature then takes an intermediate value. Radiative cooling by water and the presence of hot dust strongly influence the water molecule energy level populations and therefore should be taken into account in calculating the maser effect. We find that for a small slab thickness $H$ the inversion appears because of the de Jong (1973) mechanism, while for large $H$, the maser can be pumped by radiation from the dust, whose temperature differs from that of the gas.

The maser strength depends on the combination of dust types. When the medium is optically thick to the line radiation, 
the inversion of the $6_{16} \rightarrow 5_{23}$ maser level populations appears only for combinations of silicates with carbon (or graphite). The main cycle of maser pumping is $5_{23} \stackrel{5.5 \mu \mathrm{m}}{\longrightarrow} 6_{34}^{\mathrm{E}} \stackrel{7.6 \mu \mathrm{m}}{\longrightarrow}$ $7_{43} \stackrel{21 \mu \mathrm{m}}{\longrightarrow} 6_{16}$. The upward transition in this cycle is dominated by radiation from the hot dust, while the heat sink is realized by photon absorption by the cold dust. Combinations of water ice with any other dust type produces no inversion because of the gap in the ice absorption coefficient near $30 \mu \mathrm{m}$. Thus, masers operating on the difference between the gas and ice temperatures (Deguchi 1981; BP04) are extinguished by the hot dust radiation.

Strong masers can also be produced if there is a size distribution of the dust grains. The maser effect appears due to the de Jong mechanism for all discussed dust types, if slab halfthickness $H \lesssim 10^{15} \mathrm{~cm}$. We find that the maser disappears at $H \lesssim 10^{11} \mathrm{~cm}$, because the gas temperature becomes too low due to water cooling. For graphite, the inversion exists also at $H \gtrsim 10^{15} \mathrm{~cm}$, where the maser pumping cycle appears to be the same as in the two-dust maser model.

We show that the hot-cold dust model is able to reproduce the strength of water masers observed from a disk around the companion of the carbon star in the binary system V778 Cyg. However, the masers in the winds of AGB stars require an additional source of heating, for example due to friction between drifting dust grains and the gas.

Acknowledgements. This work was supported by the Magnus Ehrnrooth Foundation, the Finnish Graduate School for Astronomy and Space Physics (N.B.), and the Academy of Finland (J.P.). We are grateful to Dmitrii Nagirner for the code computing $K$ - and $L$-functions, Ryszard Szczerba for providing dust absorption coefficients and Seppo Alanko for useful discussions.

\section{References}

Babkovskaia, N., \& Poutanen, J. 2004, A\&A, 418, 117 (BP04)

Bains, I., Cohen, R. J., Louridas, A., et al. 2003, MNRAS, 342, 8

Bertie, J. E., Labbe, H. J., \& Whalley, E. 1969, J. Chem. Phys., 50, 4501

Bolgova, G. T., Strelnitskii, V. S., \& Shmeld, I. K. 1977, Soviet Astronomy, 21, 468

Chandra, S., Kegel, W. H., Varshalovich, D. A., \& Albrecht, M. A. 1984a, A\&A, 140, 295

Chandra, S., Varshalovich, D. A., \& Kegel, W. H. 1984b, A\&AS, 55, 51

Collison, A. J., \& Watson, W. D. 1995, ApJ, 452, L103

Cooke, B., \& Elitzur, M. 1985, ApJ, 295, 175
Danchi, W. C., Bester, M., Degiacomi, C. G., Greenhill, L. J., \& Townes, C. H. 1994, AJ, 107, 1469

David, P., \& Pegourie, B. 1995, A\&A, 293, 833

de Jong, T. 1973, A\&A, 26, 297

de Jong, T. 1977, A\&A, 55, 137

Deguchi, S. 1977, PASJ, 29, 669

Deguchi, S. 1981, ApJ, 249, 145

Downes, D., Genzel, R., Becklin, E. E., \& Wynn-Williams, C. G. 1981, ApJ, 244, 869

Elitzur, M. 1991, Astronomical Masers (Dordrecht: Kluwer Academic Publishers)

Engels, D. 1994, A\&A, 285, 497

Engels, D., \& Leinert, C. 1994, A\&A, 282, 858

Goldreich, P., \& Kwan, J. 1974, ApJ, 191, 93

Goldreich, P., \& Scoville, N. 1976, ApJ, 205, 144

Goldsmith, P. F., \& Langer, W. D. 1978, ApJ, 222, 881

Green, S. 1980, ApJS, 42, 103

Green, S., Maluendes, S., \& McLean, A. D. 1993, ApJS, 85, 181

Groenewegen, M. A. T. 1994, A\&A, 290, 531

Hartquist, T. W., Dalgarno, A., \& Oppenheimer, M. 1980, ApJ, 236, 182

Hollenbach, D., \& McKee, C. F. 1979, ApJS, 41, 555

Hudgins, D. M., Sandford, S. A., Allamandola, L. J., \& Tielens, A. G. G. M. 1993, ApJS, 86, 713

Humphreys, E. M. L., Yates, J. A., Gray, M. D., Field, D., \& Bowen, G. H. 2001, A\&A, 379, 501

Jeong, K. S., Winters, J. M., Le Bertre, T., \& Sedlmayr, E. 2003, A\&A, 407, 191

Jura, M. 1996, ApJ, 472, 806

Kegel, W. H. 1975, A\&A, 44, 95

Laor, A., \& Draine, B. T. 1993, ApJ, 402, 441

Mathis, J. S., Rumpl, W., \& Nordsieck, K. H. 1977, ApJ, 217, 425

Neufeld, D. A., Lepp, S., \& Melnick, G. J. 1995, ApJS, 100, 132

Neufeld, D. A., \& Melnick, G. J. 1987, ApJ, 322, 266

Richards, A. M. S., Yates, J. A., \& Cohen, R. J. 1999, MNRAS, 306, 954

Rosen, B. R., Moran, J. M., Reid, M. J., et al. 1978, ApJ, 222, 132

Rouleau, F., \& Martin, P. G. 1991, ApJ, 377, 526

Shakura, N. I., \& Sunyaev, R. A. 1973, A\&A, 24, 337

Sobolev, V. V. 1960, Moving envelopes of stars (Cambridge: Harvard University Press)

Strelnitskij, V. S. 1977, Soviet Astronomy, 21, 381

Szczerba, R., Szymczak, M., Babkovskaia, N., et al. 2005, A\&A, submitted [arXiv: astro-ph/0504354]

Tielens, A. G. G. M., \& Hollenbach, D. 1985, ApJ, 291, 722

Toth, R. A. 1991, J. Opt. Soc. Am. B, 8, 2236

Wallin, B. K., \& Watson, W. D. 1997, ApJ, 476, 685

Yamamura, I., Dominik, C., de Jong, T., Waters, L. B. F. M., \& Molster, F. J. 2000, A\&A, 363, 629

Yates, J. A., Field, D., \& Gray, M. D. 1997, MNRAS, 285, 303 